\title{
WEAK COMPACTNESS AND NO PARTIAL SQUARES
}

\author{
JOHN KRUEGER
}

\begin{abstract}
We present a characterization of weakly compact cardinals in terms of generalized stationarity. We apply this characterization to construct a model with no partial square sequences.
\end{abstract}

One of the striking features of weak compactness in the large cardinal hierarchy is the wide variety of characterizations of the concept. These characterizations meet many disparate areas of set theory, including metamathematics, combinatorics, and elementary embeddings, among others. In this paper we expand on this theme by characterizing weak compactness in terms of generalized stationarity.

The property of Mahloness illustrates a way to define or characterize a large cardinal property: specify a naturally defined set, and then assert that the large cardinal property holds iff the set is stationary. For example, a cardinal $\kappa$ is Mahlo iff the set of strongly inaccessible cardinals below $\kappa$ is stationary.

The first goal of the paper is to characterize weakly compact cardinals along the same lines. Let $\kappa>\omega$ be a regular cardinal. Let $S$ be the set of $N$ in $P_{\kappa}\left(H\left(\kappa^{+}\right)\right)$ satisfying: (1) $N \cap \kappa$ is strongly inaccessible, (2) $N^{<N \cap \kappa} \subseteq N$, and (3) the transitive collapse of $N$ is a 1-elementary substructure of $H\left((N \cap \kappa)^{+}\right)$. We will prove that $\kappa$ is weakly compact iff $S$ is stationary in $P_{\kappa}\left(H\left(\kappa^{+}\right)\right)$.

The second goal of the paper is to demonstrate how this characterization of weak compactness can be applied in forcing arguments. The property that a forcing poset $\mathbb{P}$ is $\lambda$-distributive, for a regular cardinal $\lambda$, is $\Pi_{1}$ over $H\left(\delta^{+}\right)$, where $\mathbb{P} \in H\left(\delta^{+}\right)$ and $\lambda \leq \delta^{+}$, and thus this property is preserved under 1-elementarity. This fact will enable us to use the above characterization of weak compactness to prove that a certain forcing iteration is distributive.

Specifically, we would like to construct a model in which there are no partial square sequences on any stationary subset of $\mu^{+} \cap \operatorname{cof}(\mu)$, where $\mu$ is a regular uncountable cardinal. A partial square sequence on a set $A \subseteq \mu^{+} \cap \operatorname{cof}(\mu)$ is a sequence $\left\langle c_{\alpha}: \alpha \in A\right\rangle$ satisfying that each $c_{\alpha}$ is a club subset of $\alpha$ with order type $\mu$, and if $\gamma$ is a common limit point of $c_{\alpha}$ and $c_{\beta}$, then $c_{\alpha} \cap \gamma=c_{\beta} \cap \gamma$. If there is such a sequence with domain $A$, we say that $A$ carries a partial square. It is known to be consistent that there are no stationary subsets of $\mu^{+} \cap \operatorname{cof}(\mu)$ which carry a partial square sequence. This follows from a strong stationary set reflection property which holds in a model of Magidor [4].

We would like to construct a model with no partial squares more directly. Specifically, we iterate forcing to destroy the stationarity of any subset of $\mu^{+} \cap \operatorname{cof}(\mu)$ which carries a partial square. In general, such an iteration will collapse cardinals. So first we prepare the ground model by Lévy collapsing a weakly compact cardinal $\kappa$ to become $\mu^{+}$. Then we use the above characterization of weak compactness to show that the iteration of club adding is $\kappa$-distributive and thus preserves

Date: May 2010. 
cardinals. The proof that the iteration is distributive demonstrates how to use elementary substructures, as an alternative to elementary embeddings, in applications of weak compactness.

\section{Preliminaries and Notation}

We review some basic ideas and notation which will be important in the paper.

Let $N$ be a set satisfying extensionality: if $x$ and $y$ are distinct elements of $N$, then there is $z$ in $N$ such that $z \in x \backslash y$ or $z \in y \backslash x$. Define a function $\pi$ with domain $N$ by $\in$-recursion:

$$
\pi(x)=\{\pi(y): y \in x\} .
$$

Let $\bar{N}$ be the range of $\pi$. Then $\bar{N}$ is transitive, and

$$
\pi:(N, \in) \rightarrow(\bar{N}, \in)
$$

is an isomorphism. Moreover, if $M$ is a transitive set and $\sigma:(N, \in) \rightarrow(M, \in)$ is an isomorphism, then $M=\bar{N}$ and $\sigma=\pi$. We call $\bar{N}$ the transitive collapse of $N$, and $\pi$ the transitive collapsing map. The map $\pi$ satisfies that if $X \subseteq N$ and $X$ is transitive, then $\pi \uparrow X$ is the identity.

More generally, let $E$ be a relation on a set $X$. We say $E$ is well-founded if whenever $A$ is a non-empty subset of $X$, then there is $a \in A$ which is $E$-minimal in $A$, meaning that there is no $y$ in $A$ such that $y E a$. We say $E$ is extensional if whenever $x$ and $y$ are distinct elements of $X$, there is $z$ in $X$ such that $z E x$ and $\neg z E y$, or $z E y$ and $\neg z E x$.

Suppose $E$ is a well-founded, extensional relation on a set $X$. Define a function $\pi$ with domain $X$ by $E$-recursion:

$$
\pi(x)=\{\pi(y): y E x\} .
$$

Then the range of $\pi, \pi[X]$, is a transitive set, and

$$
\pi:(X, E) \rightarrow(\pi[X], \in)
$$

is an isomorphism. Moreover, if $M$ is a transitive set and $\sigma:(X, E) \rightarrow(M, \in)$ is an isomorphism, then $M=\pi[X]$ and $\sigma=\pi$. Again we call $\pi[X]$ the transitive collapse of $(X, E)$, and $\pi$ the transitive collapsing map.

We will be concerned with stationary subsets of

$$
P_{\kappa}\left(H\left(\kappa^{+}\right)\right)=\left\{a \subseteq H\left(\kappa^{+}\right):|a|<\kappa\right\},
$$

where $\kappa$ is a regular uncountable cardinal. Recall that a set $C \subseteq P_{\kappa}\left(H\left(\kappa^{+}\right)\right)$is $c l u b$ if (1) whenever $\left\langle a_{i}: i\langle\zeta\rangle\right.$ is a $\subseteq$-increasing sequence of sets in $C$, where $\zeta<\kappa$, then $\bigcup_{i<\zeta} a_{i} \in C$, and (2) for all $a$ in $P_{\kappa}\left(H\left(\kappa^{+}\right)\right)$, there is $b$ in $C$ such that $a \subseteq b$. A set $S \subseteq P_{\kappa}\left(H\left(\kappa^{+}\right)\right)$is stationary if $S \cap C \neq \emptyset$, for every club set $C \subseteq P_{\kappa}\left(H\left(\kappa^{+}\right)\right)$. We will use the following characterization of stationarity. Let $S$ be a subset of $\left\{a \in P_{\kappa}\left(H\left(\kappa^{+}\right)\right): a \cap \kappa \in \kappa\right\}$. Then $S$ is stationary iff for every function $F: H\left(\kappa^{+}\right)^{<\omega} \rightarrow H\left(\kappa^{+}\right)$, there is $a$ in $S$ such that $F\left[a^{<\omega}\right] \subseteq a$.

Let $\mathbb{P}$ be a forcing poset, and $\lambda$ an ordinal. A $\mathbb{P}$-name $\dot{X}$ is a nice name for a subset of $\lambda$ if every element of $\dot{X}$ is an ordered pair $\langle p, \check{\xi}\rangle$, where $\xi \in \lambda$, and for each $\xi$ in $\lambda$, the set

$$
\{p:\langle p, \check{\xi}\rangle \in \dot{X}\}
$$

is an antichain of $\mathbb{P}$. If $p$ is a condition in $\mathbb{P}$ and $p \Vdash \dot{Y} \subseteq \check{\lambda}$, then there is a nice name $\dot{X}$ for a subset of $\lambda$ such that $p \Vdash \dot{X}=\dot{Y}$. 


\section{Some $\Pi_{1}^{1}$ Expressions}

In this section we describe some properties of models of the form $\left(V_{\alpha}, \in\right)$, using $\Pi_{1}^{1}$-formulas. These expressions will be used in the next section to show that if $\kappa$ is $\Pi_{1}^{1}$-indescribable, then there are stationary many $N$ in $P_{\kappa}\left(H\left(\kappa^{+}\right)\right)$such that the transitive collapse of $N$ is a 1-elementary substructure of $H\left((N \cap \kappa)^{+}\right)$.

We begin by specifying a second-order language $\mathcal{L}$. The non-logical symbols of $\mathcal{L}$ are: (1) a binary relation symbol $\in,(2)$ unary predicate symbols $\dot{\alpha}, \dot{E}, \dot{K}$, $\dot{T}$, and $\dot{C}$. First order variables will always be written in lower case, and second order variables will always be written in upper case. If $\Phi$ is a formula of $\mathcal{L}$, we write $\Phi\left(X_{1}, \ldots, X_{n}, x_{1}, \ldots, x_{k}\right)$ to indicate that the free variables of $\Phi$ are among $X_{1}, \ldots X_{n}, x_{1}, \ldots, x_{k}$.

Let $\Delta$ denote the set of formulas of $\mathcal{L}$ which have no second order quantifications. We say a formula of $\mathcal{L}$ is $\Pi_{1}^{1}$ if it is of the form $\forall X_{1} \cdots \forall X_{n} \Psi$, where $\Psi$ is in $\Delta$. Any conjunction of $\Pi_{1}^{1}$ formulas is logically equivalent to a $\Pi_{1}^{1}$ formula. We will sometimes speak loosely and refer to a formula as being $\Pi_{1}^{1}$ when we really mean it is logically equivalent to a $\Pi_{1}^{1}$ formula.

Let $\kappa$ be a strongly inaccessible cardinal. A set $M$ is said to be a $\kappa$-model if $M$ is transitive, the cardinality of $M$ is equal to $\kappa, \kappa$ is a member of $M, M^{<\kappa} \subseteq M$, and $M$ is a model of $\mathrm{ZFC}^{-}$(that is, the theory ZFC minus the power-set axiom). Note that any $\kappa$-model is a subset of $H\left(\kappa^{+}\right)$. Also, if $M \prec H\left(\kappa^{+}\right), \kappa+1 \subseteq M, M$ has size $\kappa$, and $M^{<\kappa} \subseteq M$, then $M$ is a $\kappa$-model.

Let us briefly describe the intended interpretation of the non-logical symbols of $\mathcal{L}$. Suppose $\kappa$ is strongly inaccessible, $M$ is a $\kappa$-model, and $H: M^{<\omega} \rightarrow M$ is a function. We code the structure $(M, \in, H)$ as a subset of $V_{\kappa}$ as follows. Fix a bijection $\pi: \kappa \rightarrow M$. Define $E \subseteq \kappa \times \kappa$ by letting $x E y$ if $\pi(x) \in \pi(y)$. Define $K: \kappa^{<\omega} \rightarrow \kappa$ by letting $K\left(\beta_{1}, \ldots, \beta_{n}\right)=\pi^{-1}\left(H\left(\pi\left(\beta_{1}\right), \ldots, \pi\left(\beta_{n}\right)\right)\right)$. Clearly $\pi$ : $(\kappa, E, K) \rightarrow(M, \in, H)$ is an isomorphism. Now interpret the symbols $\dot{\alpha}, \dot{E}$, and $\dot{K}$ by the sets $\kappa, E$, and $K$ respectively. We interpret the predicate symbol $\dot{T}$ as a subset of $V_{\kappa}$ which codes the elementary diagram of the structure $(\kappa, E, K)$. We interpret $\dot{C}$ as a particular club subset of $\kappa$.

Let us recall the following basic fact about the satisfaction relation. Suppose $\Phi\left(X_{1}, \ldots, X_{n}\right)$ is a formula of $\mathcal{L}$ which does not mention certain non-logical symbols of $\mathcal{L}$. Then the satisfaction of this formula in a model $\left(V_{\kappa}, \in, \kappa, E, K, T, C\right)$ is equivalent to its satisfaction in any restriction of this model obtained by omitting the interpretations of some of the predicate symbols which do not occur in $\Phi$.

We begin our description of some $\Pi_{1}^{1}$ properties by reviewing the well-known fact that the statements " $\alpha$ is strongly inaccessible" and " $M$ is an $\alpha$-model" are $\Pi_{1}^{1}$.

Lemma 2.1. There is a $\Pi_{1}^{1}$ sentence $\Phi_{0}$ such that for any ordinal $\alpha,\left(V_{\alpha}, \in\right) \models \Phi_{0}$ iff $\alpha$ is strongly inaccessible.

Proof. Let $\Phi_{0}$ be the conjunction of the following sentences:

(1) For every ordinal $\beta, \beta+1$ exists.

(2) There exists a limit ordinal.

(3) For every ordinal $\beta$, there is an ordinal $\gamma$ and a function from $\gamma$ onto the power set of $\beta$.

(4) For all $X$, if $X$ is an ordinal-valued operation with domain an ordinal, then the range of $X$ is bounded.

Note that (1), (2), and (3) are in $\Delta$, and (4) is $\Pi_{1}^{1}$. 
Let LST denote the set of Gödel numbers for formulas in the first order language of set theory, and let $\mathrm{ZFC}^{-}$be the subset of LST consisting of the axioms of ZFC minus the power set axiom.

We will consider structures which are expansions of $\left(V_{\alpha}, \in, \alpha, E\right)$, where $E \subseteq$ $\alpha \times \alpha$. We would like to express statements of the form " $(\alpha, E)$ satisfies such and such formulas of LST" using the language $\mathcal{L}$. To this end, we review the idea of a truth assignment.

Let $\Gamma(Z)$ be the conjunction of the following formulas of $\mathcal{L}$ :

- $Z$ is an operation, taking values 0 and 1 , defined on pairs $(\varphi, h)$, where $\varphi \in \operatorname{LST}$ and $h$ is a function which assigns to each variable of LST a member of $\dot{\alpha}$.

- $Z(x=y, h)=0$ iff $h(x)=h(y)$.

- $Z(x \in y, h)=0$ iff $h(x) \dot{E} h(y)$.

- $Z(\varphi \wedge \psi, h)=0$ iff $Z(\varphi, h)=0$ and $Z(\psi, h)=0$.

- $Z(\neg \varphi, h)=0$ iff $Z(\varphi, h)=1$.

- $Z(\exists x \varphi, h)=0$ iff there is $b$ in $\dot{\alpha}$ such that $Z\left(\varphi, h^{\prime}\right)=0$, where $h^{\prime}$ is the function equal to $h$ everywhere except at $x$, and $h^{\prime}(x)=b$.

Note that the formula $\Gamma$ is in $\Delta$. If $T \subseteq V_{\alpha}$, then $\left(V_{\alpha}, \in, \alpha, E\right) \models \Gamma[T]$ iff $T$ is a truth assignment for the structure $(\alpha, E)$ in the usual sense, namely, that for every formula $\varphi\left(x_{1}, \ldots, x_{n}\right)$ of $\operatorname{LST}$ and $a_{1}, \ldots, a_{n}$ in $\alpha, T(\varphi, h)=0$ iff $(\alpha, E) \models$ $\varphi\left[a_{1}, \ldots, a_{n}\right]$, where $h$ is any function satisfying that $h\left(x_{i}\right)=a_{i}$ for $i=1, \ldots, n$.

For example, we can express the statement

$$
\left(V_{\alpha}, \in, \alpha, E\right) \models "(\alpha, E) \models \varphi\left[a_{1}, \ldots, a_{n}\right] "
$$

to mean that for any $T \subseteq V_{\alpha}$ such that

$$
\left(V_{\alpha}, \in, \alpha, E\right) \models \Gamma[T],
$$

$T(\varphi, h)=0$ for any function $h$ satisfying that $h\left(x_{i}\right)=a_{i}$ for all $i=1, \ldots, n$. Clearly this is expressible by a $\Pi_{1}^{1}$ formula of $\mathcal{L}$.

We sometimes write $T\left(\varphi, a_{1}, \ldots, a_{n}\right)=0$ to abbreviate that the free variables of $\varphi$ are included among $x_{1}, \ldots, x_{n}$, and $T(\varphi, h)=0$ for some (any) function $h$ which assigns values $a_{1}, \ldots, a_{n}$ to the variables $x_{1}, \ldots, x_{n}$. When $\varphi$ is a sentence of LST, we will sometimes write $T(\varphi)$ to mean $T(\varphi, h)$ for some (any) $h$.

There are straightforward variations on these expressions which we will use. For example, we could write down a similar $\Delta$ formula with free variables $X$ and $Z$ which assert that $X \subseteq \dot{\alpha} \times \dot{\alpha}$ and $Z$ is a truth assignment for $(\dot{\alpha}, X)$. We will also consider truth assignments for models expanding $(\alpha, E)$.

When writing down formulas of $\mathcal{L}$, we will sometimes write $x \dot{E} y$ to abbreviate $\dot{E}(\langle x, y\rangle)$.

Lemma 2.2. There is a $\Pi_{1}^{1}$ sentence $\Phi_{1}$ satisfying the following. Suppose $\alpha$ is strongly inaccessible and $E \subseteq V_{\alpha}$. Then $\left(V_{\alpha}, \in, \alpha, E\right) \models \Phi_{1}$ iff $E \subseteq \alpha \times \alpha,(\alpha, E)$ is well-founded and extensional, and the transitive collapse of $(\alpha, E)$ is an $\alpha$-model.

Proof. Let $\Phi_{1}$ be the conjunction of the following sentences:

(1) For all $x$, if $\dot{E}(x)$, then $x$ is an ordered pair of ordinals.

(2) For every ordinal-valued function $f$ with domain $\omega$, there is $n<\omega$ such that $\neg(f(n+1) \dot{E} f(n))$. 
(3) For any distinct ordinals $x$ and $y$, there is $z$ such that either $(z \dot{E} x \wedge \neg(z \dot{E} y))$ or $(z \dot{E} y \wedge \neg(z \dot{E} x))$.

(4) For every truth assignment $Y$ for the structure $(\dot{\alpha}, \dot{E})$, for every $\varphi$ in $\mathrm{ZFC}^{-}$, $Y(\varphi)=0$.

(5) There is $x$ in $\dot{\alpha}$ such that $(\dot{\alpha}, \dot{E}) \models$ " $x$ is an ordinal", and for all $y$ in $\dot{\alpha}$ there is $z>y$ in $\dot{\alpha}$ such that $z \dot{E} x$.

(6) For any ordinal-valued function $g$ with domain an ordinal, there is $x$ in $\dot{\alpha}$ such that for all $y$ in $\dot{\alpha}, y \dot{E} x$ iff $y$ is in the range of $g$.

Note that each of the above statements is in $\Delta$, except (4) which is $\Pi_{1}^{1}$.

Suppose $\alpha$ is strongly inaccessible and $E \subseteq V_{\alpha}$. Let $\mathcal{A}=\left(V_{\alpha}, \in, \alpha, E\right)$. Then easily, $\mathcal{A}$ models (1) iff $E \subseteq \alpha \times \alpha, \mathcal{A}$ models (2) iff $(\alpha, E)$ is well-founded (this uses the fact that $\alpha$ is strongly inaccessible), and $\mathcal{A}$ models $(3)$ iff $(\alpha, E)$ is extensional. Assume that these three properties hold. Let $(M, \in)$ be the transitive collapse of $(\alpha, E)$, and let $\pi:(\alpha, E) \rightarrow(M, \in)$ be the transitive collapsing map. Then $\mathcal{A}$ models $(4)$ iff $(\alpha, E)$ is a model of $\mathrm{ZFC}^{-}$iff $(M, \in)$ is a model of $\mathrm{ZFC}^{-}$, since $(\alpha, E)$ and $(M, \in)$ are isomorphic. The structure $\mathcal{A}$ models (5) iff there is an ordinal $\xi$ in $M$ such that $\xi \geq \alpha$, which is equivalent to $\alpha$ being in $M$, since $M$ is transitive.

Suppose $\mathcal{A}$ models (6), and we show $M^{<\alpha} \subseteq M$. Let $a$ be a subset of $M$ of size less than $\alpha$, and we show $a$ is in $M$. Fix a bijection $f: \beta \rightarrow a$, where $\beta<\alpha$. Define $g: \beta \rightarrow \alpha$ by letting $g(i)=\pi^{-1}(f(i))$. Clearly $g$ is in $V_{\alpha}$, since $\beta<\alpha$ and $\alpha$ is strongly inaccessible. By (6), fix $x$ in $\alpha$ such that for all $y, y E x$ iff $y$ is in the range of $g$. Then $\pi(x)=\{\pi(y): y E x\}=\{\pi(y): y \in \operatorname{ran}(g)\}=\left\{\pi\left(\pi^{-1}(f(i))\right): i<\beta\right\}=$ $\{f(i): i<\beta\}=a$. So $\pi(x)=a$ and $a$ is in $M$.

On the other hand, assume $M^{<\alpha} \subseteq M$, and let $g: \beta \rightarrow \alpha$ be in $V_{\alpha}$. So $\beta<\alpha$. Define $f: \beta \rightarrow M$ by letting $f(i)=\pi(g(i))$. By the closure of $M, \operatorname{ran}(f)$ is in $M$. Fix $x$ in $\alpha$ such that $\pi(x)=\operatorname{ran}(f)$. Then $y E x$ iff $\pi(y) \in \pi(x)=\operatorname{ran}(f)$ iff there is $i<\beta$ such that $\pi(y)=f(i)$. But $f(i)=\pi(g(i))$ and $\pi$ is an isomorphism. So the last statement is equivalent to the statement that there is $i<\beta$ such that $y=g(i)$, which is equivalent to the statement that $y$ is in the range of $g$.

Lemma 2.3. There is a formula $\Theta(X, S)$ in $\Delta$ satisfying the following. Suppose $\alpha$ is strongly inaccessible, and $F$ and $T$ are subsets of $V_{\alpha}$. Then

$$
\left(V_{\alpha}, \in, \alpha\right) \models \Theta[F, T]
$$

iff $F \subseteq \alpha \times \alpha,(\alpha, F)$ is well-founded and extensional, the transitive collapse of $(\alpha, F)$ is an $\alpha$-model, and $T$ is a truth assignment for $(\alpha, F)$.

Proof. The proof is nearly identical to the proof of the previous lemma. Define $\Theta(S, X)$ by replacing all occurrences in $\Phi_{1}$ of an atomic formula of the form " $\dot{E}(x)$ " with the atomic formula " $x \in X$ ", and replace (4) with " $S$ is a truth assignment for $(\dot{\alpha}, X)$ and $S(\varphi)=0$ for every $\varphi$ in $\mathrm{ZFC}^{-"}$.

Our next goal is to prove that for a strongly inaccessible cardinal $\alpha$ and an $\alpha$-model $M$, the statement that $M$ is a 1-elementary substructure of $H\left(\alpha^{+}\right)$is a $\Pi_{1}^{1}$ property over $V_{\alpha}$. Specifically, let $E \subseteq \alpha \times \alpha$ be such that $(\alpha, E)$ and $(M, \in)$ are isomorphic. Then there is a $\Pi_{1}^{1}$ formula $\Phi_{2}$ such that $\left(V_{\alpha}, \in, \alpha, E\right) \models \Phi_{2}$ iff $(M, \in) \prec_{1}\left(H\left(\alpha^{+}\right), \in\right)$.

First we prove some technical lemmas which show how to express the relation $M \subseteq N$, between $\alpha$-models $M$ and $N$, with a formula in $\Delta$. 
Lemma 2.4. Suppose $\alpha$ is strongly inaccessible, $M$ and $N$ are $\alpha$-models, $E$ and $F$ are subsets of $\alpha \times \alpha$, and $(M, \in)$ and $(N, \in)$ are isomorphic to $(\alpha, E)$ and $(\alpha, F)$ respectively. Let $\pi:(\alpha, E) \rightarrow(M, \in)$ and $\sigma:(\alpha, F) \rightarrow(N, \in)$ be the transitive collapsing maps. Then $M \subseteq N$ iff there exists a function $G: \alpha \rightarrow \alpha$ such that $\sigma \circ G=\pi$.

Proof. Assume $M \subseteq N$. Define $G: \alpha \rightarrow \alpha$ by $G=\sigma^{-1} \circ \pi$. This makes sense because $M \subseteq N$. Then $\sigma \circ G=\sigma \circ \sigma^{-1} \circ \pi=\pi$. Conversely, assume $G: \alpha \rightarrow \alpha$ is a function such that $\sigma \circ G=\pi$. Then $M=\pi[\alpha]=\sigma[G[\alpha]] \subseteq \sigma[\alpha]=N$.

Lemma 2.5. Suppose $\alpha, M, N, E, F, \pi$, and $\sigma$ are as described in the assumptions of Lemma 2.4. Then for any function $G: \alpha \rightarrow \alpha$, the following statements are equivalent.

(1) $\sigma \circ G=\pi$.

(2) (a) $x E y$ iff $G(x) F G(y)$ for all $x, y$ in $\alpha$, and (b) if $z F G(x)$, then $z$ is in the range of $G$, for all $x, z$ in $\alpha$.

Proof. Suppose $G: \alpha \rightarrow \alpha$ is a function and $\sigma \circ G=\pi$. Then $M \subseteq N$, and $G=\sigma^{-1} \circ \pi$. For $x, y$ in $\alpha, x E y$ iff $\pi(x) \in \pi(y)$ (since $\pi:(\alpha, E) \rightarrow(M, \in)$ is an isomorphism) iff $G(x)=\sigma^{-1}(\pi(x)) F \sigma^{-1}(\pi(y))=G(y)$ (since $\sigma^{-1}:(N, \in) \rightarrow$ $(\alpha, F)$ is an isomorphism). So $x E y$ iff $G(x) F G(y)$, proving (a).

For (b), assume $z F G(x)$. Since $\sigma:(\alpha, F) \rightarrow(N, \in)$ is an isomorphism, $\sigma(z) \in$ $\sigma(G(x))=\pi(x)$. So $\sigma(z) \in \pi(x)$. Now $\pi(x)$ is in $M$, and $M$ is transitive, so $\sigma(z)$ is in $M$. Let $z^{\prime}$ be in $\alpha$ such that $\pi\left(z^{\prime}\right)=\sigma(z)$. Then $\sigma(z)=\pi\left(z^{\prime}\right)=\sigma\left(G\left(z^{\prime}\right)\right)$. But $\sigma$ is injective, so $z=G\left(z^{\prime}\right)$. Thus $z$ is in the range of $G$, which proves (b).

Conversely, assume $G: \alpha \rightarrow \alpha$ is a function satisfying (2). We prove $\sigma \circ G=\pi$ by $E$-induction. So let $y$ be in $\alpha$, and suppose for all $x$ such that $x E y, \sigma(G(x))=\pi(x)$. Then $\pi(y)=\{\pi(x): x E y\}=\{\sigma(G(x)): x E y\}$. By (a), this last set is equal to $\{\sigma(G(x)): G(x) F G(y)\}$. On the other hand, $\sigma(G(y))=\{\sigma(z): z F G(y)\}$. But $\{z: z F G(y)\}=\{G(x): G(x) F G(y)\}$ by (b). So $\sigma(G(y))=\{\sigma(G(x))$ : $G(x) F G(y)\}=\pi(y)$.

Lemma 2.6. There is a formula $\Psi(X, Y)$ in $\Delta$ satisfying the following. Suppose $\alpha$ is strongly inaccessible, $M$ and $N$ are $\alpha$-models, and $E$ and $F$ are subsets of $\alpha \times \alpha$ such that $(M, \in)$ and $(N, \in)$ are isomorphic to $(\alpha, E)$ and $(\alpha, F)$ respectively. Let $\pi:(\alpha, E) \rightarrow(M, \in)$ and $\sigma:(\alpha, F) \rightarrow(N, \in)$ be the transitive collapsing maps. Then for any $G \subseteq V_{\alpha},\left(V_{\alpha}, \in, \alpha, E\right) \models \Psi[F, G]$ iff $G: \alpha \rightarrow \alpha$ is a function such that $\sigma \circ G=\pi$.

Proof. Let $\Psi(X, Y)$ be the conjunction of the following formulas:

(1) $Y$ is an operation which maps ordinals to ordinals.

(2) For all $x, y$ in $\dot{\alpha}, x \dot{E} y$ iff $\langle Y(x), Y(y)\rangle \in X$.

(3) For all $x, z$ in $\dot{\alpha}$, if $\langle z, Y(x)\rangle \in X$, then there is $y$ in $\dot{\alpha}$ such that $z=Y(y)$. Clearly $\left(V_{\alpha}, \in, \alpha, E\right) \models \Psi[F, G]$ iff $G: \alpha \rightarrow \alpha$ is a function, $x E y$ iff $G(x) F G(y)$, and $z F G(x)$ implies $z$ is in the range of $G$. By Lemma 2.5, this is equivalent to the statement that $G: \alpha \rightarrow \alpha$ is a function such that $\sigma \circ G=\pi$.

Lemma 2.7. Let $\alpha$ be strongly inaccessible, and let $M$ be an $\alpha$-model. Then the following are equivalent.

(1) $(M, \in) \prec_{1}\left(H\left(\alpha^{+}\right), \in\right)$. 
(2) For every $\Delta_{0}$-formula $\varphi\left(x, x_{1}, \ldots, x_{n}\right)$ in the first order language of set theory, for all $a_{1}, \ldots, a_{n}$ in $M$, for every $\alpha$-model $N$ such that $M \subseteq N$, if there is $c \in N$ such that $(N, \in) \models \varphi\left[c, a_{1}, \ldots, a_{n}\right]$, then there is $b \in M$ such that $(M, \in) \models \varphi\left[b, a_{1}, \ldots, a_{n}\right]$.

Proof. Assume (1) holds. Let $\varphi\left(x, x_{1}, \ldots, x_{n}\right)$ be $\Delta_{0}$, let $a_{1}, \ldots, a_{n}$ be in $M$, and let $N$ be an $\alpha$-model such that $M \subseteq N$. Assume there is $c$ in $N$ such that $(N, \in) \models$ $\varphi\left[c, a_{1}, \ldots, a_{n}\right]$. Since $N$ is an $\alpha$-model, $N \subseteq H\left(\alpha^{+}\right)$. But $\varphi$ is $\Delta_{0}$, so $\left(H\left(\alpha^{+}\right), \in\right.$ )$=\varphi\left[c, a_{1}, \ldots, a_{n}\right]$. Hence $\left(H\left(\alpha^{+}\right), \in\right) \models \exists x \varphi\left[a_{1}, \ldots, a_{n}\right]$. As $M \prec_{1} H\left(\alpha^{+}\right)$, $(M, \in) \models \exists x \varphi\left[a_{1}, \ldots, a_{n}\right]$. So there is $b$ in $M$ such that $(M, \in) \models \varphi\left[b, a_{1}, \ldots, a_{n}\right]$.

Now assume $(2)$ holds. Let $\varphi\left(x, x_{1}, \ldots, x_{n}\right)$ be $\Delta_{0}$ and let $a_{1}, \ldots, a_{n}$ be in $M$. Assume $\left(H\left(\alpha^{+}\right), \in\right)=\exists x \varphi\left[a_{1}, \ldots, a_{n}\right]$. Fix $c$ such that $\left(H\left(\alpha^{+}\right), \in\right) \models \varphi\left[c, a_{1}, \ldots, a_{n}\right]$. Since $\alpha^{<\alpha}=\alpha$, we can find an $\alpha$-model $N$ such that $M \cup\{c\} \subseteq N$. As $\varphi$ is $\Delta_{0},(N, \in$ )$\models \varphi\left[c, a_{1}, \ldots, a_{n}\right]$. By $(2)$, there is $b$ in $M$ such that $(M, \in) \models \varphi\left[b, a_{1}, \ldots, a_{n}\right]$. So $(M, \in) \models \exists x \varphi\left[a_{1}, \ldots, a_{n}\right]$.

Proposition 2.8. There is a $\Pi_{1}^{1}$ sentence $\Phi_{2}$ satisfying the following. Suppose $\alpha$ is strongly inaccessible, $M$ is an $\alpha$-model, $E \subseteq \alpha \times \alpha$, and $(\alpha, E)$ is isomorphic to $(M, \in)$. Then $\left(V_{\alpha}, \in, \alpha, E\right) \models \Phi_{2}$ iff $(M, \in) \prec_{1}\left(H\left(\alpha^{+}\right), \in\right)$.

Proof. Let $\Theta(X, S)$ and $\Psi(X, Y)$ be the formulas from Lemmas 2.3 and 2.6. Let $\Psi^{*}\left(X, Y, S, S^{\prime}\right)$ be the formula: $S^{\prime}$ is a truth assignment for $(\dot{\alpha}, \dot{E}), \Theta(X, S)$, and $\Psi(X, Y)$. Note that $\Psi^{*}$ is in $\Delta$.

Let $\Phi_{2}$ be the following sentence: for all $X, Y, S, S^{\prime}$, for every $\Delta_{0}$ formula $\varphi\left(x, x_{1}, \ldots, x_{n}\right)$ in LST, and for all $a_{1}, \ldots, a_{n}$ in $\dot{\alpha}$ : either $\neg \Psi^{*}$, or $\left(\Psi^{*}\right.$, and if there exists $c$ in $\dot{\alpha}$ such that $S\left(\varphi, c, Y\left(a_{1}\right), \ldots, Y\left(a_{n}\right)\right)=0$, then there exists $b$ in $\dot{\alpha}$ such that $\left.S^{\prime}\left(\varphi, b, a_{1}, \ldots, a_{n}\right)=0\right)$. Clearly $\Phi_{2}$ is $\Pi_{1}^{1}$.

Let $\alpha$ be strongly inaccessible, and assume $M$ is an $\alpha$-model. Let $E$ be a subset of $\alpha \times \alpha$ such that $(M, \in)$ is isomorphic to $(\alpha, E)$, and let $\pi:(\alpha, E) \rightarrow(M, \in)$ be the transitive collapsing map.

Assume $\left(V_{\alpha}, \in, \alpha, E\right) \models \Phi_{2}$. We verify $(M, \in) \prec_{1}\left(H\left(\alpha^{+}\right), \in\right)$ by proving (2) of Lemma 2.7. So let $\varphi\left(x, x_{1}, \ldots, x_{n}\right)$ be $\Delta_{0}$, and let $a_{1}, \ldots, a_{n}$ be in $M$. Suppose $N$ is an $\alpha$-model, $M \subseteq N, c \in N$, and $(N, \in) \models \varphi\left[c, a_{1}, \ldots, a_{n}\right]$. We show there is $b$ in $M$ such that $(M, \in) \models \varphi\left[b, a_{1}, \ldots, a_{n}\right]$.

Fix $F \subseteq \alpha \times \alpha$ such that $(\alpha, F)$ is isomorphic to $(N, \in)$. Let $T$ and $T^{\prime}$ be truth assignments for the structures $(\alpha, F)$ and $(\alpha, E)$ respectively. Let $\sigma:(\alpha, F) \rightarrow(N, \in)$ be the transitive collapsing map. Since $M \subseteq N$, by Lemma 2.4 there is a function $G: \alpha \rightarrow \alpha$ satisfying $\sigma \circ G=\pi$. It follows that $\left(V_{\alpha}, \in, \alpha, E\right) \models \Psi^{*}\left[F, G, T, T^{\prime}\right]$. Now $c \in N$ and $(N, \in) \models \varphi\left[c, a_{1}, \ldots, a_{n}\right]$. Since $\sigma^{-1}:(N, \in) \rightarrow(\alpha, F)$ is an isomorphism, $(\alpha, F)=\varphi\left[\sigma^{-1}(c), \sigma^{-1}\left(a_{1}\right), \ldots, \sigma^{-1}\left(a_{n}\right)\right]$.

For each $i=1, \ldots, n, a_{i}$ is in $M$, so fix $\gamma_{i}$ in $\alpha$ such that $\pi\left(\gamma_{i}\right)=a_{i}$. Since $\sigma \circ G=$ $\pi, \sigma\left(G\left(\gamma_{i}\right)\right)=a_{i}$. So $\sigma^{-1}\left(a_{i}\right)=G\left(\gamma_{i}\right)$. Hence $(\alpha, F) \models \varphi\left[\sigma^{-1}(c), G\left(\gamma_{1}\right), \ldots, G\left(\gamma_{n}\right)\right]$. So $T\left(\varphi, \sigma^{-1}(c), G\left(\gamma_{1}\right), \ldots, G\left(\gamma_{n}\right)\right)=0$.

Since $\left(V_{\alpha}, \in, \alpha, E\right) \models \Phi_{2}$, there is $b$ in $\alpha$ such that $T^{\prime}\left(\varphi, b, \gamma_{1}, \ldots, \gamma_{n}\right)=0$. Therefore $(\alpha, E) \models \varphi\left[b, \gamma_{1}, \ldots, \gamma_{n}\right]$. As $\pi:(\alpha, E) \rightarrow(M, \in)$ is an isomorphism, $(M, \in) \models \varphi\left[\pi(b), \pi\left(\gamma_{1}\right), \ldots, \pi\left(\gamma_{n}\right)\right]$, that is, $(M, \in) \models \varphi\left[\pi(b), a_{1}, \ldots, a_{n}\right]$. This proves that $(M, \in) \prec_{1}\left(H\left(\alpha^{+}\right), \in\right)$.

On the other hand, assume $(M, \in) \prec_{1}\left(H\left(\alpha^{+}\right), \in\right)$, and we show $\left(V_{\alpha}, \in, \alpha, E\right) \models$ $\Phi_{2}$. So let $F, G, T, T^{\prime}$ be subsets of $V_{\alpha}, \varphi\left(x, x_{1}, \ldots, x_{n}\right)$ a $\Delta_{0}$-formula, and $a_{1}, \ldots, a_{n}$ in $\alpha$. Suppose $\left(V_{\alpha}, \in, \alpha, E\right) \models \Psi^{*}\left[F, G, T, T^{\prime}\right]$. By the choice of $\Theta, F \subseteq \alpha \times \alpha,(\alpha, F)$ 
is well-founded and extensional, the transitive collapse of $(\alpha, F)$ is an $\alpha$-model, and $T$ is a truth assignment for $(\alpha, F)$. Let $(N, \in)$ be the transitive collapse of $(\alpha, F)$, and let $\sigma:(\alpha, F) \rightarrow(N, \in)$ be the transitive collapsing map. By the choice of $\Psi$, $G: \alpha \rightarrow \alpha$ is a function and $\sigma \circ G=\pi$. Also $T^{\prime}$ is a truth assignment for $(\alpha, E)$.

Suppose there exists $c$ in $\alpha$ such that $T\left(\varphi, c, G\left(a_{1}\right), \ldots, G\left(a_{n}\right)\right)=0$. Then $(\alpha, F)=\varphi\left[c, G\left(a_{1}\right), \ldots, G\left(a_{n}\right)\right]$. We need to show there is $b$ in $\alpha$ such that $T^{\prime}\left(\varphi, b, a_{1}, \ldots, a_{n}\right)=0$, that is, $(\alpha, E) \models \varphi\left[b, a_{1}, \ldots, a_{n}\right]$. Since $\sigma:(\alpha, F) \rightarrow(N, \in)$ is an isomorphism, $(N, \in) \models \varphi\left[\sigma(c), \sigma\left(G\left(a_{1}\right)\right), \ldots, \sigma\left(G\left(a_{n}\right)\right)\right]$. As $\sigma \circ G=\pi$, $(N, \in) \models \varphi\left[\sigma(c), \pi\left(a_{1}\right), \ldots, \pi\left(a_{n}\right)\right]$. Now $\varphi$ is $\Delta_{0}$ and $N \subseteq H\left(\alpha^{+}\right)$, so $\left(H\left(\alpha^{+}\right), \in\right.$ )$\models \varphi\left[\sigma(c), \pi\left(a_{1}\right), \ldots, \pi\left(a_{n}\right)\right]$. Since $(M, \in) \prec_{1}\left(H\left(\alpha^{+}\right), \in\right)$ and $M=\pi[\alpha]$, there is $b$ in $\alpha$ such that $(M, \in) \models \varphi\left[\pi(b), \pi\left(a_{1}\right), \ldots, \pi\left(a_{n}\right)\right]$. As $\pi:(\alpha, E) \rightarrow(M, \in)$ is an isomorphism, $(\alpha, E) \models \varphi\left[b, a_{1}, \ldots, a_{n}\right]$.

\section{Weak Compactness and Generalized Stationarity}

With the $\Pi_{1}^{1}$ expressions from the last section at hand, we are now ready to prove our generalized stationarity characterization of weak compactness. Let $\mathcal{L}$ be the second order language from the previous section. If $\kappa$ is weakly compact, then $\kappa$ is $\Pi_{1}^{1}$-indescribable. In our context, this means that if $E, K, T$, and $C$ are subsets of $V_{\kappa}$, and $\Phi$ is a $\Pi_{1}^{1}$ sentence of $\mathcal{L}$ such that $\left(V_{\kappa}, \in, \kappa, E, K, T, C\right) \models \Phi$, then there is $\alpha<\kappa$ such that $\left(V_{\alpha}, \in, \alpha, E \cap V_{\alpha}, K \cap V_{\alpha}, T \cap V_{\alpha}, C \cap V_{\alpha}\right) \models \Phi$.

For easy reference, we describe again the three $\Pi_{1}^{1}$-sentences $\Phi_{0}, \Phi_{1}$, and $\Phi_{2}$ introduced in the last section.

For any ordinal $\alpha,\left(V_{\alpha}, \in\right) \models \Phi_{0}$ iff $\alpha$ is strongly inaccessible.

Suppose $\alpha$ is strongly inaccessible and $E \subseteq V_{\alpha}$. Then $\left(V_{\alpha}, \in, \alpha, E\right) \models \Phi_{1}$ iff $E \subseteq \alpha \times \alpha,(\alpha, E)$ is well-founded and extensional, and the transitive collapse of $(\alpha, E)$ is an $\alpha$-model.

Suppose $\alpha$ is strongly inaccessible, $M$ is an $\alpha$-model, $E \subseteq \alpha \times \alpha$, and $(M, \in)$ is isomorphic to $(\alpha, E)$. Then $\left(V_{\alpha}, \in, \alpha, E\right) \models \Phi_{2}$ iff $(M, \in) \prec_{1}\left(H\left(\alpha^{+}\right), \in\right)$.

For a regular cardinal $\kappa$, if $N$ is in $P_{\kappa}\left(H\left(\kappa^{+}\right)\right)$, we write $\kappa_{N}$ for $N \cap \kappa$.

Theorem 3.1. Let $\kappa$ be a regular cardinal. Let $S$ be the set of $N$ in $P_{\kappa}\left(H\left(\kappa^{+}\right)\right)$ satisfying:

(1) $\kappa_{N}$ is strongly inaccessible,

(2) $N^{<\kappa_{N}} \subseteq N$

(3) $(\bar{N}, \in) \prec_{1}\left(H\left(\kappa_{N}^{+}\right), \in\right)$.

Then $\kappa$ is weakly compact iff $S$ is stationary.

Proof. Suppose $\kappa$ is weakly compact. Then $\kappa$ is $\Pi_{1}^{1}$-indescribable. Let

$$
F: H\left(\kappa^{+}\right)^{<\omega} \rightarrow H\left(\kappa^{+}\right)
$$

be a function. We will find a set $N$ in $S$ which is closed under $F$. Since $\kappa$ is strongly inaccessible, $\kappa^{<\kappa}=\kappa$, so we can find a $\kappa$-model $M$ which is an elementary substructure of $H\left(\kappa^{+}\right)$and is closed under $F$. Let

$$
H=F \uparrow M^{<\omega} \text {. }
$$

Let us code the structure $(M, \in, H)$ as a subset of $V_{\kappa}$. Fix a bijection $\pi$ : $\kappa \rightarrow M$. Define a relation $E$ on $\kappa$ by letting $x E y$ if $\pi(x) \in \pi(y)$. Then $\pi$ : $(\kappa, E) \rightarrow(M, \in)$ is an isomorphism. It follows that $(\kappa, E)$ is well-founded and 
extensional. Since $M$ is transitive, $M$ is the transitive collapse of $(\kappa, E)$, and $\pi$ is the transitive collapsing map. Define an operation $K: \kappa^{<\omega} \rightarrow \kappa$ by letting $K\left(\xi_{1}, \ldots, \xi_{n}\right)=\pi^{-1}\left(H\left(\pi\left(\xi_{1}\right), \ldots, \pi\left(\xi_{n}\right)\right)\right)$. Then clearly

$$
\pi:(\kappa, E, K) \rightarrow(M, \in, H)
$$

is an isomorphism.

Next we interpret the non-logical predicate symbols of $\mathcal{L}$. We use $\kappa, E$, and $K$ to interpret the symbols $\dot{\alpha}, \dot{E}$, and $\dot{K}$.

We interpret the symbol $\dot{T}$ with a truth predicate $T$ for the structure $(\kappa, E, K)$. Let $L^{+}$be the set of Gödel numbers for formulas in the first order language of set theory, augmented with one function symbol. Let $T$ be the set of finite sequences of the form $\left\langle\varphi, \xi_{1}, \ldots, \xi_{n}\right\rangle$, where $\varphi$ is a formula in $L^{+}$with free variables $x_{1}, \ldots, x_{n}$, $\xi_{1}, \ldots, \xi_{n}$ are in $\kappa$, and $(\kappa, E, K) \models \varphi\left[\xi_{1}, \ldots, \xi_{n}\right]$.

Let $C$ be the club set of $\alpha$ in $\kappa$ such that:

(1) for all $y$ in $\alpha$, if $|\{x: x E y\}|<\kappa$, then $\{x: x E y\} \subseteq \alpha$,

(2) for all $\xi_{1}, \ldots, \xi_{n}$ in $\alpha, K\left(\xi_{1}, \ldots, \xi_{n}\right) \in \alpha$.

We interpret the symbol $\dot{C}$ with $C$.

Let $\mathcal{A}$ be the structure

$$
\left(V_{\kappa}, \in, \kappa, E, K, T, C\right)
$$

in the language $\mathcal{L}$.

Now we consider some $\Pi_{1}^{1}$ sentences satisfied by $\mathcal{A}$. Since $\kappa$ is strongly inaccessible, $\mathcal{A}=\Phi_{0}$. As $(\kappa, E)$ is well-founded and extensional, and the transitive collapse of $(\kappa, E)$ is the $\kappa$-model $(M, \in), \mathcal{A}=\Phi_{1}$. Since $(M, \in) \prec\left(H\left(\kappa^{+}\right), \in\right)$, in particular, $(M, \in) \prec_{1}\left(H\left(\kappa^{+}\right), \in\right)$. So $\mathcal{A} \models \Phi_{2}$.

Let us define two more sentences $\Phi_{3}$ and $\Phi_{4}$ of $\mathcal{L}$. Let $\Phi_{3}$ be the sentence: for every truth assignment $S$ for the structure $(\dot{\alpha}, \dot{E}, \dot{K})$, for every formula $\varphi\left(x_{1}, \ldots, x_{n}\right)$ of $L^{+}$, and for all ordinals $\xi_{1}, \ldots, \xi_{n},\left\langle\varphi, \xi_{1}, \ldots, \xi_{n}\right\rangle$ is in $T$ iff $S\left(\varphi, \xi_{1}, \ldots, \xi_{n}\right)=0$. Note that $\Phi_{3}$ is $\Pi_{1}^{1}$, and $\mathcal{A} \models \Phi_{3}$.

Let $\Phi_{4}$ be the sentence: for every ordinal $\beta$, there is an ordinal $\gamma>\beta$ which is in $\dot{C}$. Since $C$ is club in $\kappa, \mathcal{A}=\Phi_{4}$. Note that $\Phi_{4}$ is in $\Delta$.

Now we apply the $\Pi_{1}^{1}$-indescribability of $\kappa$. We have that

$$
\left(V_{\kappa}, \in, \kappa, E, K, T, C\right) \models \Phi_{0} \wedge \Phi_{1} \wedge \Phi_{2} \wedge \Phi_{3} \wedge \Phi_{4} .
$$

By $\Pi_{1}^{1}$-indescribability, fix an ordinal $\alpha<\kappa$ such that

$$
\left(V_{\alpha}, \in, \alpha, E \cap V_{\alpha}, K \cap V_{\alpha}, T \cap V_{\alpha}, C \cap V_{\alpha}\right) \models \Phi_{0} \wedge \Phi_{1} \wedge \Phi_{2} \wedge \Phi_{3} \wedge \Phi_{4} .
$$

Let $E_{\alpha}=E \cap V_{\alpha}$ and $K_{\alpha}=K \cap V_{\alpha}$. Note that by the choice of $\Phi_{4}, \alpha$ is a limit point of $C$, so $\alpha$ is in $C$. In particular, $\alpha$ is closed under $K$, so $K_{\alpha}=K\left\lceil\alpha^{<\omega}\right.$. Let $T_{\alpha}=T \cap V_{\alpha}$. Note that $C \cap V_{\alpha}=C \cap \alpha$. Let

$$
\mathcal{B}=\left(V_{\alpha}, \in, \alpha, E_{\alpha}, K_{\alpha}, T_{\alpha}, C \cap \alpha\right) .
$$

Let us draw some relevant conclusions from the choice of $\alpha$. Since $\mathcal{B} \models \Phi_{0}, \alpha$ is strongly inaccessible. Since $\mathcal{B} \models \Phi_{1}, E_{\alpha} \subseteq \alpha \times \alpha,\left(\alpha, E_{\alpha}\right)$ is well-founded and extensional, and the transitive collapse of $\left(\alpha, E_{\alpha}\right)$ is an $\alpha$-model. Let $\left(M_{\alpha}, \in\right)$ be the transitive collapse of $\left(\alpha, E_{\alpha}\right)$, and let $\pi_{\alpha}:\left(\alpha, E_{\alpha}\right) \rightarrow\left(M_{\alpha}, \in\right)$ be the transitive collapsing map. Since $\mathcal{B} \models \Phi_{2},\left(M_{\alpha}, \in\right) \prec_{1}\left(H\left(\alpha^{+}\right), \in\right)$. 
Since $\mathcal{B}=\Phi_{3}$, for every formula $\varphi\left(x_{1}, \ldots, x_{n}\right)$ in $L^{+}$, and for all $\xi_{1}, \ldots, \xi_{n}$ in $\alpha$, $\left(\alpha, E_{\alpha}, K_{\alpha}\right)=\varphi\left[\xi_{1}, \ldots, \xi_{n}\right]$ iff $\left\langle\varphi, \xi_{1}, \ldots, \xi_{n}\right\rangle \in T_{\alpha}$. But this is true iff $\left\langle\varphi, \xi_{1}, \ldots, \xi_{n}\right\rangle$ is in $T$, which by definition of $T$ is equivalent to $(\kappa, E, K) \models \varphi\left[\xi_{1}, \ldots, \xi_{n}\right]$. It follows that $\left(\alpha, E_{\alpha}, K_{\alpha}\right)$ is an elementary substructure of $(\kappa, E, K)$.

We noted above that since $\mathcal{B}=\Phi_{4}, \alpha$ is in $C$. It follows that if $y$ is in $\alpha$ and $|\{x: x E y\}|<\kappa$, then $\{x: x E y\} \subseteq \alpha$. In particular, if $|\{x: x E y\}|<\kappa$, then $\left\{x: x E_{\alpha} y\right\}=\{x: x E y\}$.

Define

$$
H_{\alpha}: M_{\alpha}^{<\omega} \rightarrow M_{\alpha}
$$

by letting $H_{\alpha}\left(d_{1}, \ldots, d_{n}\right)=\pi_{\alpha}\left(K_{\alpha}\left(\pi_{\alpha}^{-1}\left(d_{1}\right), \ldots, \pi_{\alpha}^{-1}\left(d_{n}\right)\right)\right)$. Then clearly

$$
\pi_{\alpha}:\left(\alpha, E_{\alpha}, K_{\alpha}\right) \rightarrow\left(M_{\alpha}, \in, H_{\alpha}\right)
$$

is an isomorphism.

Define a function

$$
j:\left(M_{\alpha}, \in, H_{\alpha}\right) \rightarrow(M, \in, H)
$$

by

$$
j=\pi \circ \pi_{\alpha}^{-1} .
$$

Since $\pi_{\alpha}^{-1}:\left(M_{\alpha}, \in, H_{\alpha}\right) \rightarrow\left(\alpha, E_{\alpha}, K_{\alpha}\right)$ is an isomorphism, $\left(\alpha, E_{\alpha}, K_{\alpha}\right)$ is an elementary substructure of $(\kappa, E, K)$, and $\pi:(\kappa, E, K) \rightarrow(M, \in, H)$ is an isomorphism, it follows that $j:\left(M_{\alpha}, \in, H_{\alpha}\right) \rightarrow(M, \in, H)$ is an elementary embedding.

Let $N=j\left[M_{\alpha}\right]$. Since $j$ is an elementary embedding,

$$
\left(N, \in, H \uparrow N^{<\omega}\right) \prec(M, \in, H) .
$$

In particular, $N$ is closed under $H$. Therefore $N$ is closed under $F$, since $H=F \uparrow$ $M^{<\omega}$. So we will be done if we can show that $N$ is in $S$.

First we show that the critical point of $j$ is equal to $\alpha$, and $j(\alpha)=\kappa$. Since the property of being a cardinal is $\Pi_{1}$, and $M_{\alpha} \prec_{1} H\left(\alpha^{+}\right)$, for all $\beta$ in $M_{\alpha}, \beta$ is a cardinal in $M_{\alpha}$ iff $\beta$ is cardinal in $H\left(\alpha^{+}\right)$iff $\beta$ is a cardinal. So $M_{\alpha}$ models that $\alpha$ is the largest cardinal. But $M$ models that $\kappa$ is the largest cardinal, since $M \prec H\left(\kappa^{+}\right)$. Since $j$ is elementary, $j(\alpha)=\kappa$.

We prove by induction that $j(\beta)=\beta$ for all $\beta<\alpha$. So let $\beta<\alpha$, and assume $j(\xi)=\xi$ for all $\xi<\beta$. Let $x=\pi_{\alpha}^{-1}(\beta)$. Then $\pi(x)=\pi\left(\pi_{\alpha}^{-1}(\beta)\right)=j(\beta)$, and $\pi_{\alpha}(x)=\pi_{\alpha}\left(\pi_{\alpha}^{-1}(\beta)\right)=\beta$. So it suffices to show $\pi(x)=\pi_{\alpha}(x)$. Since $\beta<\alpha$, $\pi(x)=j(\beta)<j(\alpha)=\kappa$. So $\pi(x)<\kappa$. But $\pi(x)=\{\pi(y): y E x\}$, so the set $\{y: y E x\}$ has size less than $\kappa$. As $\alpha$ is in $C,\{y: y E x\}=\left\{y: y E_{\alpha} x\right\}$. If $y E_{\alpha} x$, then $\pi_{\alpha}(y) \in \pi_{\alpha}(x)=\beta$, so by induction, $j\left(\pi_{\alpha}(y)\right)=\pi_{\alpha}(y)$. But $j\left(\pi_{\alpha}(y)\right)=$ $\pi\left(\pi_{\alpha}^{-1}\left(\pi_{\alpha}(y)\right)\right)=\pi(y)$. So $\pi(y)=\pi_{\alpha}(y)$. Hence $j(\beta)=\pi(x)=\{\pi(y): y E x\}=$ $\left\{\pi_{\alpha}(y): y E_{\alpha} x\right\}=\pi_{\alpha}(x)=\beta$.

It follows easily that $N \cap \kappa=\alpha$. On the one hand, if $\beta<\alpha$, then $\beta=j(\beta) \in$ $j\left[M_{\alpha}\right]=N$, and $\beta=j(\beta)<j(\alpha)=\kappa$. Thus $\alpha \subseteq N \cap \kappa$. On the other hand, suppose $\gamma$ is in $N \cap \kappa$, and fix $\beta \in M_{\alpha}$ such that $j(\beta)=\gamma$. Then $j(\beta)=\gamma<\kappa=j(\alpha)$, so $\beta<\alpha$. So $\beta=j(\beta)=\gamma$, and $\gamma$ is in $\alpha$.

Finally, we verify that $N$ is in $S$. The set $N$ has size equal to $\left|M_{\alpha}\right|$, which is $\alpha$. And $\kappa_{N}=N \cap \kappa=\alpha$, which is strongly inaccessible. Suppose $a$ is a subset of $N$ of size less than $\kappa_{N}$. Then $j^{-1}[a]$ is a subset of $M_{\alpha}$ of size less than $\kappa_{N}$. Since $M_{\alpha}$ is an $\alpha$-model, $j^{-1}[a]$ is in $M_{\alpha}$. Therefore $j\left(j^{-1}[a]\right)$ is in $N$. But as $|a|<\kappa_{N}$, 
and $\kappa_{N}$ is the critical point of $j, j\left(j^{-1}[a]\right)=j\left[j^{-1}[a]\right]=a$. So $a$ is in $N$. Finally, since $N$ is isomorphic to $M_{\alpha}$, and $M_{\alpha}$ is transitive, $N$ is equal to $M_{\alpha}$. Therefore $(\bar{N}, \in) \prec_{1}\left(H\left(\kappa_{N}\right), \in\right)$.

Now we prove the other direction of the theorem. Assume $S$ is stationary in $P_{\kappa}\left(H\left(\kappa^{+}\right)\right)$. We prove that $\kappa$ is $\Pi_{1}^{1}$-indescribable.

First note that by (1) in the definition of $S$, the set of strongly inaccessible cardinals below $\kappa$ is stationary. So $\kappa$ is Mahlo. In particular, $\left|V_{\kappa}\right|=\kappa$, so $V_{\kappa} \in$ $H\left(\kappa^{+}\right)$.

Consider the second order language of set theory with one predicate symbol. Let $R$ be a subset of $V_{\kappa}$, and let $\Phi(X)$ be a formula in this language containing no second order quantifiers. Assume $\left(V_{\kappa}, \in, R\right) \models \forall X \Phi$. This is equivalent to

$$
\forall A \subseteq V_{\kappa}\left(V_{\kappa}, \in, R\right) \models \Phi[A],
$$

which is a first order statement in $\left(H\left(\kappa^{+}\right), \in\right)$.

We find $\alpha<\kappa$ such that $\left(V_{\alpha}, \in, R \cap V_{\alpha}\right) \models \forall X \Phi$. Fix a set $N$ in $S$ such that $N \prec H\left(\kappa^{+}\right)$and $\left(V_{\kappa}, \in, R\right) \in N$. Then $\kappa_{N}$ is strongly inaccessible, $N^{<\kappa_{N}} \subseteq N$, and $(\bar{N}, \in) \prec_{1}\left(H\left(\kappa_{N}^{+}\right), \in\right)$. By the elementarity of $N, N$ models that $\left(V_{\kappa}, \in, R\right) \models \forall X \Phi$. Let $\pi: N \rightarrow \bar{N}$ be the transitive collapsing map. Note that $\pi(\kappa)=\kappa_{N}$.

Let us check that $\pi\left(V_{\kappa}\right)=V_{\kappa_{N}}$. If $\alpha<\kappa_{N}$, then $V_{\alpha} \in N$ by elementarity. But $\left|V_{\alpha}\right|<\kappa_{N}$, since $\kappa_{N}$ is strongly inaccessible, so $V_{\alpha} \subseteq N$. This proves that $V_{\kappa_{N}} \subseteq N \cap V_{\kappa}$. On the other hand, if $x$ is in $N \cap V_{\kappa}$, then by elementarity there is $\alpha<\kappa_{N}$ such that $x$ is in $V_{\alpha}$. So $V_{\kappa} \cap N=V_{\kappa_{N}}$. But $V_{\kappa_{N}}$ is a transitive subset of $N$, so $\pi\left\lceil V_{\kappa_{N}}\right.$ is the identity function. Therefore $\pi\left(V_{\kappa}\right)=\left\{\pi(x): x \in N \cap V_{\kappa}\right\}=$ $\left\{\pi(x): x \in V_{\kappa_{N}}\right\}=\left\{x: x \in V_{\kappa_{N}}\right\}=V_{\kappa_{N}}$. Also, since $N \cap V_{\kappa}=V_{\kappa_{N}}$, it follows that $R \cap N=R \cap V_{\kappa_{N}}$, and hence $\pi(R)=\{\pi(x): x \in R \cap N\}=\left\{x: x \in R \cap V_{\kappa_{N}}\right\}=$ $R \cap V_{\kappa_{N}}$.

Since $\pi$ is an isomorphism,

$$
(\bar{N}, \in) \models \pi\left(\left(V_{\kappa}, \in, R\right)\right) \models \forall X \Phi .
$$

By the last paragraph, it follows that

$$
(\bar{N}, \in) \models\left(V_{\kappa_{N}}, \in, R \cap V_{\kappa_{N}}\right) \models \forall X \Phi .
$$

This is equivalent to

$$
(\bar{N}, \in) \models \forall A \subseteq V_{\kappa_{N}}\left(V_{\kappa_{N}}, \in, R \cap V_{\kappa_{N}}\right) \models \Phi[A] .
$$

The above statement satisfied by $(\bar{N}, \in)$ is $\Pi_{1}$. But $(\bar{N}, \in) \prec_{1}\left(H\left(\kappa_{N}^{+}\right), \in\right)$. Hence

$$
\left(H\left(\kappa_{N}^{+}\right), \in\right) \models \forall A \subseteq V_{\kappa_{N}}\left(V_{\kappa_{N}}, \in, R \cap V_{\kappa_{N}}\right) \models \Phi[A] .
$$

But $H\left(\kappa_{N}^{+}\right)$contains every subset of $V_{\kappa_{N}}$, so it is correct in computing the truth of the above statement. Therefore $\left(V_{\kappa_{N}}, \in, R \cap V_{\kappa_{N}}\right) \models \forall X \Phi$.

The proof of the forward direction of the last theorem is similar to proofs that the $\Pi_{1}^{1}$-indescribability of $\kappa$ implies the existence of elementary embeddings with critical point $\kappa$ defined on $\kappa$-models; see [3]. 


\section{A REMARK ON SUBCOMPACTNESS}

We give an example of a large cardinal property, much stronger than weak compactness, which can be characterized in terms of generalized stationarity in a way surprisingly similar to the characterization of weak compactness given above.

A cardinal $\kappa$ is said to be subcompact if for every set $B \subseteq H\left(\kappa^{+}\right)$, there is a cardinal $\alpha<\kappa$, a set $A \subseteq H\left(\alpha^{+}\right)$, and an elementary embedding

$$
j:\left(H\left(\alpha^{+}\right), \in, A\right) \rightarrow\left(H\left(\kappa^{+}\right), \in, B\right)
$$

with critical point $\alpha$. Note that by elementarity, $j(\alpha)=\kappa$. Subcompact cardinals were introduced by Jensen. Schimmerling and Zeman have studied the impact of subcompact cardinals in inner model theory ([6]). Subcompactness lies between superstrong and supercompact in the large cardinal hierarchy.

The next result provides a generalized stationarity type characterization of subcompactness, which displays subcompactness as a natural strengthening of weak compactness.

Theorem 4.1. Let $\kappa$ be a regular cardinal. Let $T$ be the set of $N$ in $P_{\kappa}\left(H\left(\kappa^{+}\right)\right)$ satisfying:

(1) $\kappa_{N}$ is strongly inaccessible,

(2) $\bar{N}$ is equal to $H\left(\kappa_{N}^{+}\right)$.

Then $\kappa$ is subcompact iff $T$ is stationary.

Proof. Suppose $\kappa$ is subcompact. Let $F: H\left(\kappa^{+}\right)^{<\omega} \rightarrow H\left(\kappa^{+}\right)$be a function. We find a set $N$ in $T$ which is closed under $F$. Since $F \subseteq H\left(\kappa^{+}\right)$, there is a cardinal $\alpha<\kappa$, a set $G \subseteq H\left(\alpha^{+}\right)$, and an elementary embedding

$$
j:\left(H\left(\alpha^{+}\right), \in, G\right) \rightarrow\left(H\left(\kappa^{+}\right), \in, F\right)
$$

with critical point $\alpha$. Since $\kappa$ is strongly inaccessible and $j(\alpha)=\kappa, \alpha$ is strongly inaccessible by elementarity.

Let $N=j\left[H\left(\alpha^{+}\right)\right]$. Then $N$ is an elementary substructure of $\left(H\left(\kappa^{+}\right), \in, F\right)$. In particular, $N$ is closed under $F$. Since $\alpha$ is the critical point of $j$ and $j(\alpha)=\kappa$, it is easy to check that $N \cap \kappa=\alpha$. Now $H\left(\alpha^{+}\right)$is transitive and $j^{-1}: N \rightarrow H\left(\alpha^{+}\right)$ is an isomorphism, so by the uniqueness of the transitive collapse, $\bar{N}=H\left(\alpha^{+}\right)$. So $N$ is in $T$.

Conversely, assume $T$ is stationary, and we show $\kappa$ is subcompact. Let $B$ be a subset of $H\left(\kappa^{+}\right)$. Choose a set $N$ in $T$ such that $(N, \in, B \cap N) \prec\left(H\left(\kappa^{+}\right), \in, B\right)$. Let $\pi: N \rightarrow \bar{N}$ be the transitive collapsing map. By the definition of $T, \kappa_{N}=\pi(\kappa)$ is strongly inaccessible and $\bar{N}=H\left(\kappa_{N}^{+}\right)$. Since

$$
\pi^{-1}:\left(H\left(\kappa_{N}^{+}\right), \in, \pi[B \cap N]\right) \rightarrow(N, \in, B \cap N)
$$

is an isomorphism and $(N, \in, B \cap N) \prec\left(H\left(\kappa^{+}\right), \in, B\right)$, it follows that

$$
\pi^{-1}:\left(H\left(\kappa_{N}^{+}\right), \in, \pi[B \cap N]\right) \rightarrow\left(H\left(\kappa^{+}\right), \in, B\right)
$$

is an elementary embedding, and the critical point of $\pi^{-1}$ is $\kappa_{N}$.

\section{Partial Square Sequences}

Let $\mu$ be an uncountable cardinal. Recall that a $\square_{\mu}$-sequence is a sequence

$$
\left\langle c_{\alpha}: \alpha \in \mu^{+}, \alpha \text { limit }\right\rangle
$$


satisfying that every $c_{\alpha}$ is a club subset of $\alpha$ with order type less than or equal to $\mu$, and for every limit point $\gamma$ of $c_{\alpha}, c_{\alpha} \cap \gamma=c_{\gamma}$.

A partial square sequence is a variation of this idea, in which we require the clubs to be defined only on a set of ordinals, not necessarily on every limit ordinal. The coherence property then needs to be modified, since a limit point of a club on the sequence does not necessarily have a club attached to it. We restrict our attention to sets concentrating on a fixed cofinality; in that case, it is possible to thin out the domain and arrange that the clubs all have the same regular order type.

Definition 5.1. Let $\mu$ be an uncountable cardinal, and let $\nu \leq \mu$ be regular. Let $A \subseteq \mu^{+} \cap \operatorname{cof}(\nu)$. A sequence $\left\langle c_{\alpha}: \alpha \in A\right\rangle$ is a partial square sequence on $A$ if each $c_{\alpha}$ is a club subset of $\alpha$ with order type $\nu$, and whenever $\gamma$ is a limit point of both $c_{\alpha}$ and $c_{\beta}$, then $c_{\alpha} \cap \gamma=c_{\beta} \cap \gamma$.

We say that a set $A$ carries a partial square if $A$ is the domain of some partial square sequence. (Note that in our terminology, $A$ is not necessarily stationary.)

One of the reasons why partial square sequences are interesting is because their existence can be proven in ZFC. The main result in this direction is the following theorem of Shelah.

Theorem 5.2 (Shelah [7]). Let $\mu$ be a regular uncountable cardinal, and let $\nu<\mu$ be regular. Then $\mu^{+} \cap \operatorname{cof}(\nu)$ is the union of $\mu$ many subsets, each of which carries a partial square.

It follows from the theorem that for any stationary set $S \subseteq \mu^{+} \cap \operatorname{cof}(\nu)$, there is a club $C \subseteq \mu^{+}$such that $S \cap C$ can be partitioned into $\mu$ many stationary subsets, each of which carries a partial square.

There are two cases which are not handled by the last theoreom. First, the theorem says nothing about the existence of partial square sequences on the successor of a singular cardinal. Indeed, one of the most important open questions on this topic is whether ZFC proves the existence of a stationary subset of $\aleph_{\omega+1} \cap \operatorname{cof}\left(\omega_{1}\right)$ which carries a partial square ([2]).

The second case not handled by the theorem is whether, for an uncountable regular cardinal $\mu$, there exists a stationary subset of $\mu^{+} \cap \operatorname{cof}(\mu)$ which carries a partial square. It turns out that this is independent of ZFC. Clearly $\square_{\mu}$ implies that the set $\mu^{+} \cap \operatorname{cof}(\mu)$ itself carries a partial square. On the other hand, Magidor [4] constructed a model satisfying that for an uncountable regular cardinal $\mu$, for every stationary set $S \subseteq \mu^{+} \cap \operatorname{cof}(<\mu)$, there is a club $C \subseteq \mu^{+}$such that for all $\alpha$ in $C \cap \operatorname{cof}(\mu), S \cap \alpha$ is stationary in $\alpha$. Sakai [5] noted that this strong reflection property implies that there is no stationary subset of $\mu^{+} \cap \operatorname{cof}(\mu)$ which carries a partial square.

In the next section we will construct a model with no partial squares more directly. Specifically, we will iterate forcing to destroy the stationarity of any subset of $\mu^{+} \cap \operatorname{cof}(\mu)$ which carries a partial square. We feel this is the natural forcing for constructing a model with no partial squares. Moreover, it seems possible a similar forcing would work to resolve the problem for successors of singulars.

In general, a forcing iteration as just described will collapse cardinals. For example, this is certainly the case if $\square_{\mu}$ holds. So we first prepare the ground model by Lévy collapsing a weakly compact cardinal $\kappa$ to become $\mu^{+}$. We then use the characterization of weak compactness given above to prove the distributivity of the iteration. 
We will need the following lemma which asserts that a proper forcing poset cannot add a thread through a partial square sequence.

Lemma 5.3. Let $\mu$ be an uncountable cardinal. Let $\left\langle d_{\xi}: \xi \in \mu^{+}\right\rangle$be a sequence, where each $d_{\xi}$ is a subset of $\xi$. Suppose that $\mathbb{P}$ is a proper forcing poset. Let $G$ be a generic filter on $\mathbb{P}$. Assume that in $V[G]$, c is a club subset of $\left(\mu^{+}\right)^{V}$ which threads the sequence $\left\langle d_{\xi}: \xi \in\left(\mu^{+}\right)^{V}\right\rangle$; that is, if $\gamma$ is a limit point of $c$ below $\left(\mu^{+}\right)^{V}$, then $c \cap \gamma=d_{\gamma}$. Then $c$ is in $V$.

Proof. Suppose for a contradiction that $p$ is a condition in $\mathbb{P}$, and $p$ forces that $\dot{c}$ is a club subset of $\left(\mu^{+}\right)^{V}$, not in $V$, which threads the sequence $\left\langle d_{\xi}: \xi \in\left(\mu^{+}\right)^{V}\right\rangle$. Let $\theta$ be a sufficiently large regular cardinal, and let $N$ be a countable elementary substructure of $H(\theta)$ which contains as elements the sets $\mathbb{P}, p, \dot{c}$, and $\left\langle d_{\xi}: \xi \in \mu^{+}\right\rangle$.

Since $p$ forces that $\dot{c}$ is not in $V$, there are $q, r \leq p$ such that for some $\zeta$ in $\mu^{+}$, $q \Vdash \zeta \in \dot{c}$ and $r \Vdash \zeta \notin \dot{c}$. By the elementarity of $N$, we can choose $q, r$, and $\zeta$ in $N$. Now apply the properness of $\mathbb{P}$ to choose $N$-generic conditions $s \leq q$ and $t \leq r$.

Let $\alpha=\sup \left(N \cap \mu^{+}\right)$. So $\zeta \in \alpha$. As $s$ and $t$ are $N$-generic, $s$ and $t$ force that $\sup \left(N[\dot{G}] \cap \mu^{+}\right)$is equal to $\alpha$. Therefore as $\dot{c} \in N, s$ and $t$ force that $\alpha$ is a limit point of $\dot{c}$. Since $\dot{c}$ is forced to be a thread, $s$ and $t$ force that $\dot{c} \cap \alpha=d_{\alpha}$. Now $s \leq q$, so $s \Vdash \zeta \in \dot{c} \cap \alpha$, and thus $\zeta$ is in $d_{\alpha}$. But as $t \leq r, t \Vdash \zeta \notin \dot{c} \cap \alpha$, contradicting that $t$ forces $\dot{c} \cap \alpha=d_{\alpha}$.

\section{No Partial Squares}

Let $\kappa$ be a weakly compact cardinal, and let $\mu<\kappa$ be regular and uncountable. We will produce a generic extension in which $\kappa$ has been collapsed to become $\mu^{+}$, and there are no stationary subsets of $\mu^{+} \cap \operatorname{cof}(\mu)$ which carry a partial square sequence. Without loss of generality we also assume $2^{\kappa}=\kappa^{+}$. For if this failed, then we can precede our construction with a collapse $\operatorname{CoLL}\left(\kappa^{+}, 2^{\kappa}\right)$. This collapse does not add subsets of $\kappa$, and therefore it preserves the weak compactness of $\kappa$.

Our forcing poset will be of the form

$$
\operatorname{CoLL}(\mu,<\kappa) * \mathbb{P},
$$

where $\mathbb{P}$ is an iteration of length $\kappa^{+}$which successively destroys the stationarity of subsets of $\mu^{+} \cap \operatorname{cof}(\mu)$ which carry a partial square. Since $\operatorname{CoLL}(\mu,<\kappa)$ is $\kappa$-c.c. and has size $\kappa, \operatorname{CoLL}(\mu,<\kappa)$ forces $2^{\kappa}=\kappa^{+}$. When working in $V^{\operatorname{ColL}(\mu,<\kappa)}$, we will write $\kappa$ and $\mu^{+}$interchangeably.

By standard arguments, if $\bar{\kappa}$ is strongly inaccessible and $\mu<\bar{\kappa} \leq \kappa$, then $\operatorname{CoLL}(\mu,<\bar{\kappa})$ forces

$$
\mu^{<\mu}=\mu, 2^{\mu}=\mu^{+}, \mu^{+}=\bar{\kappa},
$$

and $\operatorname{CoLL}(\mu,<\bar{\kappa})$ is $\bar{\kappa}$-c.c. and has size $\bar{\kappa}$.

Let $\bar{\kappa}<\kappa$ be strongly inaccessible, and suppose $G$ is a generic filter on $\operatorname{CoLL}(\mu,<$ $\kappa)$. Let $G \uparrow \bar{\kappa}=\{p \uparrow(\mu \times \bar{\kappa}): p \in G\}$. Then $G \uparrow \bar{\kappa}=G \cap \operatorname{Coll}(\mu,<\bar{\kappa})$, and $G \uparrow \bar{\kappa}$ is a generic filter on $\operatorname{CoLL}(\mu,<\bar{\kappa})$.

We will use the following version of a result of Magidor.

Theorem 6.1 (Magidor [4]). Let $\bar{\kappa}<\kappa$ be strongly inaccessible. Suppose $G$ is a generic filter on $\operatorname{CoLL}(\mu,<\kappa)$. In $V[G \uparrow \bar{\kappa}]$, let $\mathbb{P}$ be a $\mu$-closed forcing poset of size less than $\kappa$. Then in $V[G]$, there is a $V[G\lceil\bar{\kappa}]$-generic filter $H$ on $\mathbb{P}$. Moreover,

$$
V[G]=V[G \uparrow \bar{\kappa}][H][K],
$$


where $K$ is a $V[G\lceil\bar{\kappa}][H]$-generic filter on some $\mu$-closed forcing poset.

In $V$ let $S$ be the set of $N$ in $P_{\kappa}\left(H\left(\kappa^{+}\right)\right)$satisfying:

- $N \prec H\left(\kappa^{+}\right)$,

- $\mu+1 \subseteq N$,

- $\kappa_{N}$ is strongly inaccessible,

- $N^{<\kappa_{N}} \subseteq N$,

- $\bar{N} \prec_{1} \bar{H}\left(\kappa_{N}^{+}\right)$

(Recall that $\kappa_{N}$ denotes $N \cap \kappa$.) Since $\kappa$ is weakly compact, the set $S$ is stationary by Theorem 3.1.

Let us prove several lemmas which analyze the interaction between sets in $S$ and the Lévy collapse.

Note that if $N$ is in $S$ and $\pi: N \rightarrow \bar{N}$ is the transitive collapsing map, then

$$
\pi(\operatorname{CoLL}(\mu,<\kappa))=\operatorname{CoLL}\left(\mu,<\kappa_{N}\right) .
$$

This is true because, since $N^{<\kappa_{N}} \subseteq N$,

$$
N \cap \operatorname{CoLL}(\mu,<\kappa)=\operatorname{CoLL}\left(\mu,<\kappa_{N}\right),
$$

and $\pi$ is the identity on this set.

Lemma 6.2. Let $N$ be in $S$, and suppose $G^{*}$ is a generic filter on $\operatorname{CoLL}\left(\mu,<\kappa_{N}\right)$. Then $\bar{N}\left[G^{*}\right] \prec_{1} H\left(\kappa_{N}^{+}\right)^{V\left[G^{*}\right]}$.

Proof. This is a standard fact which follows from the assumption that $\bar{N} \prec_{1} H\left(\kappa_{N}^{+}\right)$. For a proof, see Theorem 2.11 in Chapter 3 of [8].

Lemma 6.3. Let $N$ be in $S$, and suppose $G$ is a generic filter on $\operatorname{CoLL}(\mu,<\kappa)$. Then $N[G] \cap V=N$, and $N[G]^{<\mu} \subseteq N[G]$.

Proof. Suppose $a$ is in $N[G] \cap V$. Let $\dot{a}$ be a name for $a$ in $N$. Define $A$ as the set of $x$ such that for some $p$ in $\operatorname{CoLL}(\mu,<\kappa), p \Vdash \dot{a}=\check{x}$. Note that since $\dot{a}$ is in $N$, $A$ is in $N$ by elementarity, and as $\operatorname{CoLL}(\mu,<\kappa)$ is $\kappa$-c.c., $|A|<\kappa$. Hence $|A|<\kappa_{N}$ by elementarity, so $A \subseteq N$. But $\dot{a}^{G}=a$ is in $A$, and hence $a$ is in $N$. (This is a standard proper forcing type argument.)

Let $f: \nu \rightarrow N[G]$ be a function, where $\nu<\mu$. We show $f$ is in $N[G]$. Define $g: \nu \rightarrow N$ by letting $g(i)=\dot{a}_{i}$ be a name in $N$ such that $\dot{a}_{i}^{G}=f(i)$. Since $\operatorname{ColL}(\mu,<\kappa)$ is $\mu$-closed, $g$ is in $V$. But then in $V, g$ is in $N^{<\kappa_{N}}$, since $\mu<\kappa_{N}$, so $g$ is in $N$. As $g$ and $G$ are in $N[G]$ and $f$ is definable from $g$ and $G, f$ is in $N[G]$.

Lemma 6.4. Let $N$ be in $S$, and suppose $G$ is a generic filter on $\operatorname{CoLL}(\mu,<\kappa)$. Then

$$
\overline{N[G]}=\bar{N}\left[G \uparrow \kappa_{N}\right] .
$$

In particular, $\overline{N[G]}$ is a member of $V\left[G\left\lceil\kappa_{N}\right]\right.$, and

$$
\overline{N[G]} \prec_{1} H\left(\kappa_{N}^{+}\right)^{V\left[G\left\lceil\kappa_{N}\right]\right.} .
$$

Proof. Let $\pi: N \rightarrow \bar{N}$ and $\sigma: N[G] \rightarrow \overline{N[G]}$ be the transitive collapsing maps. We claim that $\sigma \mid N=\pi$. So let $x$ be in $N$, and suppose for all $y$ in $x$, if $y$ is in $N$ then $\sigma(y)=\pi(y)$. Since $N[G] \cap V=N$,

$$
x \cap N[G]=x \cap N .
$$


So $\sigma(x)=\{\sigma(y): y \in x \cap N[G]\}=\{\sigma(y): y \in x \cap N\}=\{\pi(y): y \in x \cap N\}=\pi(x)$. Thus $\sigma \mid N=\pi$. In particular, $\bar{N} \subseteq \overline{N[G]}$.

Next we claim that

$$
\sigma(G)=G\left\lceil\kappa_{N}\right.
$$

and in particular, $G \uparrow \kappa_{N}$ is in $\overline{N[G]}$. Since $\operatorname{CoLL}\left(\mu,<\kappa_{N}\right) \subseteq V_{\kappa_{N}}$, and $V_{\kappa_{N}}$ is a transitive subset of $N[G], \sigma$ is the identity function on $\operatorname{CoLL}\left(\mu,<\kappa_{N}\right)$. Also

$$
N[G] \cap \operatorname{CoLL}(\mu,<\kappa)=N \cap \operatorname{CoLL}(\mu,<\kappa),
$$

as $N[G] \cap V=N$. It follows that

$$
N[G] \cap \operatorname{CoLL}(\mu,<\kappa)=\operatorname{CoLL}\left(\mu,<\kappa_{N}\right) .
$$

Therefore

$$
N[G] \cap G=G\left\lceil\kappa_{N} .\right.
$$

For if $p$ is in $N[G] \cap G$, then $p$ is in $G \cap \operatorname{CoLL}\left(\mu,<\kappa_{N}\right)=G\left\lceil\kappa_{N}\right.$. On the other hand, if $p$ is in $G\left\lceil\kappa_{N}\right.$, then $p$ is in $G$ and $p$ is in $N$, since $\operatorname{CoLL}\left(\mu,<\kappa_{N}\right) \subseteq N$. So $p$ is in $N[G] \cap G$. Hence $\sigma(G)=\{\sigma(p): p \in N[G] \cap G\}=\left\{\sigma(p): p \in G\left\lceil\kappa_{N}\right\}=\right.$ $\left\{p: p \in G \nmid \kappa_{N}\right\}=G\left\lceil\kappa_{N}\right.$.

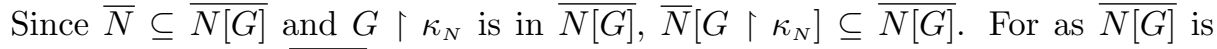
isomorphic to $N[G], \overline{N[G]}$ is closed under the interpretation function $\dot{a} \mapsto \dot{a}^{G \uparrow \kappa_{N}}$. On the other hand, suppose $a$ is in $N[G]$, and we show $\sigma(a)$ is in $\bar{N}\left[G\left\lceil\kappa_{N}\right]\right.$. Fix $\dot{a}$ in $N$ such that $\dot{a}^{G}=a$. Then $\sigma(a)=\sigma\left(\dot{a}^{G}\right)=\sigma(\dot{a})^{\sigma(G)}=\pi(\dot{a})^{G \uparrow \kappa_{N}}$, which is in $\bar{N}\left[G\left\lceil\kappa_{N}\right]\right.$.

Let us observe that the truth of several forcing properties is captured by the model $\bar{N}\left[G\left\lceil\kappa_{N}\right]\right.$, which is a 1-elementary substructure of $H\left(\kappa_{N}^{+}\right)^{V\left[G \uparrow \kappa_{N}\right]}$.

First recall that statements of the form " $p \Vdash \dot{x} \in \dot{y}$ " and " $p \Vdash \dot{x}=\dot{y}$ " are absolute between transitive sets, as can be shown by recursion on names. For example, $p \Vdash \dot{x} \in \dot{y}$ iff for all $p^{\prime} \leq p$ there is $p^{\prime \prime} \leq p^{\prime}$ and a pair $\langle s, \dot{z}\rangle$ in $\dot{y}$ such that $p^{\prime \prime} \leq s$ and $p^{\prime \prime} \Vdash \dot{x}=\dot{z}$.

Lemma 6.5. Let $\mathbb{P}$ be a forcing poset and $\lambda$ an ordinal. Then the statement that $\lambda$ is regular and $\mathbb{P}$ is $\lambda$-distributive is $\Pi_{1}$.

Proof. It is well known and easily checked that the statement " $\lambda$ is regular" is $\Pi_{1}$. The statement that $\mathbb{P}$ is $\lambda$-distributive is equivalent to the following: for all $\mathcal{D}$, for all $\beta$ in $\lambda$, and for any function $f: \beta \rightarrow \mathcal{D}$, if $f$ is surjective and every set in $\mathcal{D}$ is a dense open subset of $\mathbb{P}$, then $\bigcap \mathcal{D}$ is dense open. Recall that the property "dense open" is $\Delta_{0}$, so the last statement is $\Pi_{1}$.

Now we are ready to begin our forcing construction. We would like to iterate forcing over $V^{\operatorname{CoLL}(\mu,<\kappa)}$ to add clubs disjoint from every subset of $\kappa \cap \operatorname{cof}(\mu)$ which carries a partial square. We bookkeep so that after $\kappa^{+}$many steps, every subset of $\kappa$ has been dealt with. Actually, the forcing poset we define is not literally a forcing iteration, although it is equivalent to one.

It will be notationally convenient to use an auxiliary partially ordered set

$$
\left(X, \leq_{X}\right)
$$

which is defined in the model $V^{\operatorname{Coln}(\mu,<\kappa)}$. Let $X$ be the set of all closed, bounded subsets of $\kappa$. Let $y \leq_{X} x$ if $y$ is an end-extension of $x$, that is, if $y \cap(\max (x)+1)=x$. Note that since $2^{\mu}=\mu^{+}=\kappa$ in $V^{\operatorname{ColL}(\mu, \ll \kappa)}, X$ has size $\kappa$. 
To describe the factor forcings, let us consider the situation somewhat abstractly. Assume $\mathbb{P}$ is a $\kappa$-distributive forcing poset in $V^{\operatorname{CoLL}(\mu,<\kappa)}$. Note that since $\mathbb{P}$ is $\kappa$-distributive, every bounded subset of $\kappa$ in $V^{\operatorname{CoLL}(\mu,<\kappa) * \mathbb{P}}$ is in $V^{\operatorname{CoLL}(\mu,<\kappa)}$. In $V^{\operatorname{ColL}(\mu,<\kappa) * \mathbb{P}}$ let $A$ be a subset of $\mu^{+} \cap \operatorname{cof}(\mu)$ (we do not care whether or not $A$ is stationary). Define a forcing poset $\mathbb{P}(A)$ in $V^{\operatorname{ColL}(\mu,<\kappa) * \mathbb{P}}$ which adds a club disjoint from $A$ as follows. A condition in $\mathbb{P}(A)$ is a closed, bounded subset of $\kappa$ (that is, a member of $X$ ) which is disjoint from $A$. Let $q \leq p$ in $\mathbb{P}(A)$ if $q$ end-extends $p$ (that is, if $q \leq_{X} p$ ).

If $p$ and $q$ are conditions in $\mathbb{P}(A)$ which are compatible, then either $q \leq p$ or $p \leq q$. Indeed, assume $\max (p) \leq \max (q)$, and let $r \leq p, q$. Then $q \cap(\max (p)+1)=$ $(r \cap(\max (q)+1)) \cap(\max (p)+1)=r \cap(\max (p)+1)=p$. So $q \leq p$. In particular, every family of pairwise compatible conditions is a chain.

Lemma 6.6. Let $B$ be a chain of $\mathbb{P}(A)$ with size less than $\mu$. Let $\alpha=\sup \{\max (p)$ : $p \in B\}$. Let $q=\bigcup B \cup\{\alpha\}$. Then $q$ is in $\mathbb{P}(A)$ and $q \leq p$ for all $p \in B$.

Proof. If $B$ has a maximum element, then the maximum element is $q$, and we are done. Otherwise for all $p$ in $B, p$ is a proper initial segment of $q$. So $q$ is a condition in $\mathbb{P}(A)$ iff $\alpha$ is not in $A$. But $A$ is a subset of $\mu^{+} \cap \operatorname{cof}(\mu)$, and since $|B|<\mu$ and $B$ has no maximum element, $\alpha$ has cofinality less than $\mu$.

It follows that $\mathbb{P}(A)$ is $\mu$-closed. In general, the forcing poset $\mathbb{P}(A)$ might collapse $\kappa=\mu^{+}$. But in the specific situation we will be considering, $\mathbb{P}(A)$ will be $\kappa$ distributive.

Now we are ready to begin the description of the forcing iteration. For the remainder of the section, let $G$ be a $V$-generic filter on $\operatorname{CoLL}(\mu,<\kappa)$. Unless specified otherwise, we work in the model $V[G]$. For the purpose of bookkeeping, fix a surjective function $f: \kappa^{+} \rightarrow \kappa^{+} \times \kappa^{+}$such that $f(\alpha)=\langle i, j\rangle$ implies $i \leq \alpha$.

We define by recursion in $V[G]$ a sequence of forcing posets

$$
\left\langle\mathbb{P}_{i}: i \leq \kappa^{+}\right\rangle \text {, }
$$

and a sequence of names

$$
\left\langle\dot{T}_{j}^{i}: i, j<\kappa^{+}\right\rangle
$$

For each $\alpha<\kappa^{+}$, we write $\dot{T}(\alpha)$ for $\dot{T}_{j}^{i}$, where $f(\alpha)=\langle i, j\rangle$.

Here is a sketch of the definition. Having defined $\mathbb{P}_{\alpha}$, we define $\mathbb{P}_{\alpha+1}$ as a forcing poset which is forcing equivalent to $\mathbb{P}_{\alpha} * \mathbb{P}(\dot{T}(\alpha))$, where the second factor adds a club disjoint from $\dot{T}(\alpha)$. The forcing poset $\mathbb{P}_{\alpha}$ will force that $\dot{T}(\alpha)$ is a subset of $\mu^{+} \cap \operatorname{cof}(\mu)$ which carries a partial square. At limit stages we take a $<\kappa$-support limit. In the end, the chain condition of $\mathbb{P}_{\kappa^{+}}$will imply that any set which carries a partial square has appeared already in some previous stage, and our bookkeeping will ensure that it was made non-stationary along the way.

Before making the definition completely precise, we list our recursion hypotheses.

Recursion Hypotheses 6.7. The following statements hold for all $\alpha \leq \kappa^{+}$:

(1) If $p$ is in $\mathbb{P}_{\alpha}$, then $p: \alpha \rightarrow X$ is a partial function with $|\operatorname{dom}(p)|<\kappa$.

(2) For all $p$ and $q$ in $\mathbb{P}_{\alpha}, q \leq p$ iff $\operatorname{dom}(p) \subseteq \operatorname{dom}(q)$ and for all $i$ in $\operatorname{dom}(p)$, $q(i) \leq_{X} p(i)$.

(3) Let $\beta<\alpha$. (a) For all $q$ in $\mathbb{P}_{\alpha}, q \uparrow \beta \in \mathbb{P}_{\beta}$. (b) $\mathbb{P}_{\beta} \subseteq \mathbb{P}_{\alpha}$. (c) If $q$ is in $\mathbb{P}_{\alpha}$ and $s \leq q \uparrow \beta$ in $\mathbb{P}_{\beta}$, then letting $t=s \cup q \uparrow[\beta, \alpha), t$ is in $\mathbb{P}_{\alpha}$ and $t \leq s, q$ in $\mathbb{P}_{\alpha}$. (d) The inclusion map $\mathbb{P}_{\beta} \rightarrow \mathbb{P}_{\alpha}$ is a complete embedding. 
(4) $\mathbb{P}_{\alpha}$ is $\mu$-closed.

(5) If $\alpha<\kappa^{+}$then $\left|\mathbb{P}_{\alpha}\right|<\kappa^{+}$, and if $\alpha=\kappa^{+}$then $\mathbb{P}_{\alpha}$ is $\kappa^{+}$-c.c.

(6) If $\alpha$ is a successor ordinal $\nu+1$, then $\mathbb{P}_{\alpha}$ is forcing equivalent to $\mathbb{P}_{\nu} * \mathbb{P}(\dot{T}(\nu))$.

(7) If $\alpha<\kappa^{+}$then $\left\langle\dot{T}_{i}^{\alpha}: i<\kappa^{+}\right\rangle$is an enumeration of all nice $\mathbb{P}_{\alpha}$-names for subsets of $\kappa \cap \operatorname{cof}(\mu)$ which are forced to carry a partial square.

(8) $\mathbb{P}_{\alpha}$ is $\kappa$-distributive.

Most of the recursion hypotheses follow easily from the definition. The main challenge will be to prove $6.7(8)$, that $\mathbb{P}_{\alpha}$ is $\kappa$-distributive, and this is the only place where we will use the fact that $\kappa$ is weakly compact in $V$.

Now we make the definition of the forcing iteration precise.

Base case: Let $\mathbb{P}_{0}$ be the trivial forcing consisting of just the empty condition.

Successor case: Suppose that $\mathbb{P}_{\beta}$ is defined for all $\beta \leq \alpha$, where $\alpha<\kappa^{+}$, and moreover that sequences $\left\langle T_{i}^{\beta}: i<\kappa^{+}\right\rangle$are defined, for all $\beta<\alpha$. We assume that the recursion hypotheses 6.7 are satisfied by these objects.

Let $\left\langle\dot{T}_{i}^{\alpha}: i<\kappa^{+}\right\rangle$enumerate all nice $\mathbb{P}_{\alpha}$-names for subsets of $\mu^{+} \cap \operatorname{cof}(\mu)$ which are forced to carry a partial square. Namely, by $6.7(5),\left|\mathbb{P}_{\alpha}\right| \leq \kappa$, and since $2^{\kappa}=\kappa^{+}$, $\mathbb{P}_{\alpha}$ has at most $\kappa^{+}$many antichains. So the number of nice $\mathbb{P}_{\alpha}$-names for subsets of $\kappa=\mu^{+}$is $\left(\kappa^{+}\right)^{\kappa}=\kappa^{+}$.

Now consider the bookkeeping function $f: \kappa^{+} \rightarrow \kappa^{+} \times \kappa^{+}$. Let $f(\alpha)=\langle i, j\rangle$. Then $i \leq \alpha$. Therefore $\dot{T}_{j}^{i}=\dot{T}(\alpha)$ is defined, and $\dot{T}(\alpha)$ is a $\mathbb{P}_{i}$-name for a subset of $\mu^{+} \cap \operatorname{cof}(\mu)$ which carries a partial square. Since the inclusion map $\mathbb{P}_{i} \rightarrow \mathbb{P}_{\alpha}$ is a complete embedding and $\mathbb{P}_{\alpha}$ is $\mu$-closed, $\dot{T}(\alpha)$ is a $\mathbb{P}_{\alpha}$-name for a subset of $\mu^{+} \cap \operatorname{cof}(\mu)$. Since being a partial square sequence is upwards absolute, $\mathbb{P}_{\alpha}$ forces that $\dot{T}(\alpha)$ carries a partial square.

Let $\mathbb{P}_{\alpha+1}$ be the set of all partial functions $p: \alpha+1 \rightarrow X$ satisfying:

(1) $p\left\lceil\alpha \in \mathbb{P}_{\alpha}\right.$,

(2) if $\alpha \in \operatorname{dom}(p)$, then $p(\alpha) \in X$ and

$$
p\lceil\alpha \Vdash p(\alpha) \cap \dot{T}(\alpha)=\emptyset .
$$

Note that (2) is equivalent to

$$
p\lceil\alpha \Vdash p(\alpha) \in \mathbb{P}(\dot{T}(\alpha)) .
$$

Now define a partial ordering on $\mathbb{P}_{\alpha+1}$ by letting $q \leq p$ if

(1) $q\left\lceil\alpha \leq p\left\lceil\alpha\right.\right.$ in $\mathbb{P}_{\alpha}$,

(2) if $\alpha \in \operatorname{dom}(p)$, then $\alpha \in \operatorname{dom}(q)$ and $q(\alpha) \leq_{X} p(\alpha)$.

Limit case: Suppose $\alpha \leq \kappa^{+}$is a limit ordinal and $\mathbb{P}_{i}$ is defined for all $i<\alpha$ as required. Let $\mathbb{P}_{\alpha}$ be the set of all partial functions $p: \alpha \rightarrow X$ satisfying:

(1) $p\left\lceil i \in \mathbb{P}_{i}\right.$ for all $i<\alpha$,

(2) $|\operatorname{dom}(p)|<\kappa$.

Define a partial ordering on $\mathbb{P}_{\alpha}$ by letting $q \leq p$ in $\mathbb{P}_{\alpha}$ if $q \uparrow i \leq p \uparrow i$ in $\mathbb{P}_{i}$ for all $i<\alpha$.

Our goal is to verify the recursion hypotheses for $\mathbb{P}_{\alpha}$. Most of these are straightforward. 6.7(1) is immediate, and 6.7(2) can be easily checked. 6.7(3) is a standard fact about forcing iterations, and we omit the easy proof. 
The next lemma follows easily from $6.7(2)$.

Lemma 6.8. (1) Let $p$ and $q$ be in $\mathbb{P}_{\alpha}$, and let $\beta<\alpha$. If $q \leq p$ in $\mathbb{P}_{\alpha}$, then $q \uparrow \beta \leq p \uparrow \beta$ in $\mathbb{P}_{\beta}$.

(2) Let $\beta<\alpha$, and suppose $u$ and $t$ are in $\mathbb{P}_{\beta}$. Then $u \leq t$ in $\mathbb{P}_{\beta}$ iff $u \leq t$ in $\mathbb{P}_{\alpha}$.

The following useful fact follows almost immediately from the definition of $\mathbb{P}_{\alpha}$.

Lemma 6.9. Suppose $p: \alpha \rightarrow X$ is a partial function with $|\operatorname{dom}(p)|<\kappa$, and $p$ is not in $\mathbb{P}_{\alpha}$. Then there is $\nu<\alpha$ in $\operatorname{dom}(p)$ such that $p\left\lceil\nu \in \mathbb{P}_{\nu}\right.$, but $p\lceil\nu$ does not force that $p(\nu)$ is disjoint from $\dot{T}(\nu)$.

Lemma 6.10. Let $p$ and $q$ be in $\mathbb{P}_{\alpha}$, and suppose $p$ and $q$ are incompatible. Then there is $\nu$ in $\operatorname{dom}(p) \cap \operatorname{dom}(q)$ such that neither of $p(\nu)$ and $q(\nu)$ are initial segments of the other.

Proof. Assume that for all $\nu$ in $\operatorname{dom}(p) \cap \operatorname{dom}(q), p(\nu)$ is an initial segment of $q(\nu)$, or vice versa. We show that $p$ and $q$ are compatible. Define $r: \alpha \rightarrow X$ as the partial function with domain equal to $\operatorname{dom}(p) \cup \operatorname{dom}(q)$, such that for all $\beta$ in this domain,

$$
r(\beta)= \begin{cases}p(\beta) & \text { if } \beta \in \operatorname{dom}(p) \backslash \operatorname{dom}(q) \\ q(\beta) & \text { if } \beta \in \operatorname{dom}(q) \backslash \operatorname{dom}(p) \\ p(\beta) \cup q(\beta) & \text { if } \beta \in \operatorname{dom}(p) \cap \operatorname{dom}(q)\end{cases}
$$

It is now easy to check by cases, using Lemma 6.9, that $r$ is in $\mathbb{P}_{\alpha}$ and $r \leq p, q$.

We make a remark about notation. If $p$ is in $\mathbb{P}_{\alpha}$ and $i$ is not in the domain of $p$, then strictly speaking, $p(i)$ is not defined. However, we will find it notationally convenient to write $p(i)$ without knowing whether or not $i$ is in $\operatorname{dom}(p)$. In the case it is not, then by $p(i)$ we mean the empty set. Similarly, $\max (p(i))$ denotes the empty set if $i$ is not in $\operatorname{dom}(p)$.

The next lemma proves $6.7(4)$, that $\mathbb{P}_{\alpha}$ is $\mu$-closed.

Lemma 6.11. Suppose $B$ is a directed subset of $\mathbb{P}_{\alpha}$ of size less than $\mu$. Define a partial function $q: \alpha \rightarrow X$ with domain equal to

$$
\bigcup\{\operatorname{dom}(p): p \in B\},
$$

so that for each $\beta$ in this domain,

$$
q(\beta)=\bigcup\{p(\beta): p \in B\} \cup\{\sup \{\max (p(\beta)): p \in B\}\} .
$$

Then $q$ is in $\mathbb{P}_{\alpha}$, and $q \leq p$ for all $p \in B$.

Proof. The lemma is proved by induction. First suppose $\alpha=\xi+1$ is a successor ordinal. Then by $6.8(1),\left\{p\lceil\xi: p \in B\}\right.$ is a directed subset of $\mathbb{P}_{\xi}$ of size less than $\mu$. So by the lemma applied to $\mathbb{P}_{\xi}, q\left\lceil\xi\right.$ is in $\mathbb{P}_{\xi}$ and is below $p\lceil\xi$ for all $p \in B$. Since $B$ is directed, for all $s$ and $t$ in $B, s(\xi)$ and $t(\xi)$ are compatible with each other, and hence one is an initial segment of the other. Thus $\{p(\xi): p \in B\}$ is a chain. By Lemma 6.6 and the definition of $q(\xi), q \uparrow \xi$ forces that $q(\xi)$ is in $\mathbb{P}(\dot{T}(\xi))$ and end-extends $p(\xi)$ for all $p \in B$. It follows that $q$ is in $\mathbb{P}_{\alpha}$ and $q \leq p_{i}$ for all $i<\nu$.

Now suppose $\alpha$ is a limit ordinal. Then for all $\beta<\alpha, q \uparrow \beta$ is in $\mathbb{P}_{\beta}$ and is below $p\left\lceil\beta\right.$ for all $p \in B$, by the lemma applied to $\mathbb{P}_{\beta}$. Since $q$ has domain of size less than $\kappa, q$ is in $\mathbb{P}_{\alpha}$, and $q \leq p$ for all $p \in B$. 
Now we prove 6.7(5). Suppose $\alpha<\kappa^{+}$, and we show $\left|\mathbb{P}_{\alpha}\right|<\kappa^{+}$. The set $X$, which is the collection of closed, bounded subsets of $\kappa$, has size equal to $\kappa^{<\kappa}=\kappa$. By 6.7(1), if $p$ is in $\mathbb{P}_{\alpha}$, then $p: \alpha \rightarrow X$ is a partial function with domain of size less than $\kappa$. There are $\alpha^{<\kappa} \leq \kappa$ many possible domains, and for each choice of a domain, there are no more than $|X|^{<\kappa}=\kappa^{<\kappa}=\kappa$ many possible conditions. So $\mathbb{P}_{\alpha}$ has size at most $\kappa \times \kappa=\kappa$.

Lemma 6.12. The forcing poset $\mathbb{P}_{\kappa^{+}}$is $\kappa^{+}$-c.c.

Proof. The proof is standard. Given a family $\left\{p_{i}: i<\kappa^{+}\right\}$of conditions in $\mathbb{P}_{\kappa^{+}}$, using the fact that $\kappa^{<\kappa}=\kappa$ and the $\Delta$-system lemma, we can find an unbounded set $Z \subseteq \kappa^{+}$and a set $a \subseteq \kappa^{+}$such that for all $i<j$ in $Z, \operatorname{dom}\left(p_{i}\right) \cap \operatorname{dom}\left(p_{j}\right)=a$ and $p_{i} \uparrow a=p_{j} \uparrow a$. Let $i<j$ be in $Z$. Then Lemma 6.10 implies that $p_{i}$ and $p_{j}$ are compatible.

For 6.7(6), assume that $\alpha=\nu+1$ is a successor ordinal, and we prove that $\mathbb{P}_{\alpha}$ is forcing equivalent to $\mathbb{P}_{\nu} * \mathbb{P}(\dot{T}(\nu))$. If $p$ is in $\mathbb{P}_{\alpha}$, then by definition, $p\left\lceil\nu\right.$ is in $\mathbb{P}_{\nu}$, and $p(\nu)$ is either empty or $p \nmid \nu \Vdash p(\nu) \in \mathbb{P}(\dot{T}(\nu))$. Hence we can define a map

$$
i: \mathbb{P}_{\alpha} \rightarrow \mathbb{P}_{\nu} * \mathbb{P}(\dot{T}(\nu))
$$

by letting

$$
i(p)=p\lceil\nu * p(\nu) .
$$

Lemma 6.13. The map $i: \mathbb{P}_{\nu+1} \rightarrow \mathbb{P}_{\nu} * \mathbb{P}(\dot{T}(\nu))$, given by $i(p)=p \uparrow \nu * p(\nu)$, is an isomorphism between $\mathbb{P}_{\nu+1}$ and a dense subset of $\mathbb{P}_{\nu} * \mathbb{P}(\dot{T}(\nu))$. Hence $\mathbb{P}_{\nu+1}$ and $\mathbb{P}_{\nu} * \mathbb{P}(\dot{T}(\nu))$ are forcing equivalent.

Proof. It is straightforward to show that $i$ is an isomorphism between its domain and its range, because the orderings are determined by the end-extension relation $\leq_{X}$, which is absolute. So we only need to see that the range of $i$ is dense in $\mathbb{P}_{\nu} * \mathbb{P}(\dot{T}(\nu))$.

Suppose $s * \dot{t}$ is in $\mathbb{P}_{\nu} * \mathbb{P}(\dot{T}(\nu))$. Without loss of generality, assume $s \Vdash \dot{t} \neq \emptyset$, for otherwise we can extend to a condition which satisfies this. Since $\mathbb{P}_{\nu}$ is $\kappa$ distributive by 6.7(8), we can find $s^{\prime} \leq s$ in $\mathbb{P}_{\nu}$ and $x$ in $X$ such that $s^{\prime} \Vdash \dot{t}=\check{x}$. Define

Then $u$ is in $\mathbb{P}_{\nu+1}$ and $i(u)=s^{\prime} * \check{x} \leq s * \dot{t}$.

$$
u=s^{\prime} \cup\{\langle\nu, x\rangle\} .
$$

This completes the proof of $6.7(6) .6 .7(7)$ is immediate.

It remains to show $6.7(8)$, that $\mathbb{P}_{\alpha}$ is $\kappa$-distributive. For $\alpha=\kappa^{+}$, this follows from the recursion hypotheses.

Lemma 6.14. Suppose $\mathbb{P}_{\alpha}$ is $\kappa$-distributive for all $\alpha<\kappa^{+}$. Then $\mathbb{P}_{\kappa^{+}}$is $\kappa$ distributive.

Proof. It is straightforward to check that $\mathbb{P}_{\kappa^{+}}$is separative (this uses the fact that every set in $X$ is non-empty). Hence $\mathbb{P}_{\kappa^{+}}$is $\kappa$-distributive iff every function $f: \mu \rightarrow$ $O n$ in a generic extension by $\mathbb{P}_{\kappa^{+}}$is in the ground model.

Let $\dot{f}$ be a name for $f$. For each $i<\mu$, let $A_{i}$ be a maximal antichain contained in the dense set of conditions which decide the value of $\dot{f}(i)$. As $_{\mathbb{P}_{\kappa^{+}}}$is $\kappa^{+}$-c.c., $\left|A_{i}\right|<\kappa^{+}$for all $i<\mu$. But $\mathbb{P}_{\kappa^{+}}=\bigcup_{i<\kappa^{+}} \mathbb{P}_{i}$. So there is $\beta<\kappa^{+}$such that for all $i<\mu, A_{i} \subseteq \mathbb{P}_{\beta}$. It follows that $f$ is in the generic extension by $\mathbb{P}_{\beta}$. But $\mathbb{P}_{\beta}$ is $\kappa$-distributive by assumption, so $f$ is in the ground model. 
It remains to show that $\mathbb{P}_{\alpha}$ is $\kappa$-distributive, for all $\alpha<\kappa^{+}$. First we need several lemmas.

Lemma 6.15. Let $\nu \leq \kappa^{+}$. Let $G_{\nu}$ be a generic filter on $\mathbb{P}_{\nu}$. If $A$ is a subset of $G_{\nu}$ in $V[G]\left[G_{\nu}\right]$ of size less than $\mu$, then $A$ has a lower bound in $G_{\nu}$.

Proof. Since $G_{\nu}$ is directed, we can find a directed set $B$ with $A \subseteq B \subseteq G_{\nu}$, also of size less than $\mu$. It suffices to find a lower bound of $B$ in $G_{\nu}$. Since $\mathbb{P}_{\nu}$ is $\mu$-closed, $B$ is in the ground model $V[G]$. So in $V[G], B$ is a directed subset of $\mathbb{P}_{\nu}$ of size less than $\mu$. Let $q$ be defined as in the statement of Lemma 6.11. Then $q$ is a lower bound of $B$.

If $q$ is not in $G_{\nu}$, then there is $s$ in $G_{\nu}$ which is incompatible with $q$. By Lemma 6.10 , this means there is $\xi$ in $\operatorname{dom}(q) \cap \operatorname{dom}(s)$ such that neither of $q(\xi)$ and $s(\xi)$ is an initial segment of the other. But for any $p$ in $B, p$ and $s$ are compatible. So if $\xi$ is in $\operatorname{dom}(p)$, then $p(\xi)$ is an initial segment of $s(\xi)$, or vice versa. If $p(\xi)$ is an initial segment of $s(\xi)$ for all $p$ in $B$, then by the definition of $q, q(\xi)$ is also. Otherwise there is $p$ in $B$ such that $s(\xi)$ is an initial segment of $p(\xi)$, and then clearly $s(\xi)$ is an initial segment of $q(\xi)$. This is a contradiction, so $q$ is indeed in $G_{\nu}$

Consider $\nu<\alpha \leq \kappa^{+}$. Let $G_{\nu}$ be a $V[G]$-generic filter on $\mathbb{P}_{\nu}$. In $V[G]\left[G_{\nu}\right]$, we define a forcing poset $\mathbb{P}_{\alpha} / \mathbb{P}_{\nu}$ as follows. The underlying set of $\mathbb{P}_{\alpha} / \mathbb{P}_{\nu}$ is $\{p \uparrow[\nu, \alpha)$ : $\left.p \in \mathbb{P}_{\alpha}\right\}$. Let $t \leq s$ in $\mathbb{P}_{\alpha} / \mathbb{P}_{\nu}$ if there is $p$ in $G_{\nu}$ such that $p \cup t \leq p \cup s$ in $\mathbb{P}_{\alpha}$. Then $\mathbb{P}_{\alpha}$ is isomorphic to a dense subset of $\mathbb{P}_{\nu} *\left(\mathbb{P}_{\alpha} / \mathbb{P}_{\nu}\right)$, by the map

$$
p \mapsto p\lceil\nu * p \uparrow[\nu, \alpha) \text {. }
$$

(See Section 5 of [1] for the details.)

Lemma 6.16. Let $\nu<\alpha \leq \kappa^{+}$. Then $\mathbb{P}_{\nu}$ forces that $\mathbb{P}_{\alpha} / \mathbb{P}_{\nu}$ is $\mu$-closed.

Proof. Let $G_{\nu}$ be a $V[G]$-generic filter on $\mathbb{P}_{\nu}$. In $V[G]\left[G_{\nu}\right]$, let $\left\langle s_{i}: i\langle\zeta\rangle\right.$ be a descending sequence of conditions in $\mathbb{P}_{\alpha} / \mathbb{P}_{\nu}$, where $\zeta<\mu$ is a limit ordinal. For each $i<j<\zeta$, choose $p_{i, j}$ in $G_{\nu}$ such that

$$
p_{i, j} \cup s_{j} \leq p_{i, j} \cup s_{i}
$$

in $\mathbb{P}_{\alpha}$. Let $A=\left\{p_{i, j}: i<j<\zeta\right\}$. Then $A \subseteq G_{\nu}$ and $|A|<\mu$, so by Lemma 6.15, $A$ has a lower bound $q$ in $G_{\nu}$. By 6.7(3c), $q \cup s_{i}$ is in $\mathbb{P}_{\alpha}$ for all $i$. By 6.7(2),

$$
q \cup s_{j} \leq q \cup s_{i}
$$

in $\mathbb{P}_{\alpha}$, for all $i<j<\zeta$.

So in $V[G],\left\langle q \cup s_{i}: i<\zeta\right\rangle$ is a descending sequence of conditions in $\mathbb{P}_{\alpha}$. By Lemma 6.11, this sequence has a lower bound $r$ satisfying that $r \uparrow \nu=q$. Let $u=r \uparrow[\nu, \alpha)$, which is in $\mathbb{P}_{\alpha} / \mathbb{P}_{\nu}$. Then

$$
r=q \cup u \leq q \cup s_{i}
$$

for all $i<\zeta$. Since $q$ is in $G_{\nu}$, this implies

$$
u \leq s_{i}
$$

in $\mathbb{P}_{\alpha} / \mathbb{P}_{\nu}$ for all $i<\zeta$.

Proposition 6.17. Let $\alpha<\kappa^{+}$. Assume that $\mathbb{P}_{\nu}$ is $\kappa$-distributive for all $\nu<\alpha$. Then $\mathbb{P}_{\alpha}$ is $\kappa$-distributive. 
JOHN KRUEGER

Proof. Assume that $\mathbb{P}_{\nu}$ is $\kappa$-distributive for all $\nu<\alpha$. We show that $\mathbb{P}_{\alpha}$ is $\kappa$ distributive. Whether $\alpha$ is a successor or a limit ordinal is not relevant. Let $\mathcal{D}$ be a family of $\mu$ many dense open subsets of $\mathbb{P}_{\alpha}$, and let $p$ be a condition in $\mathbb{P}_{\alpha}$. We find $q \leq p$ which is in $\bigcap \mathcal{D}$. Choose $\operatorname{CoLL}(\mu,<\kappa)$-names $\dot{\mathbb{P}}_{\alpha}, \dot{p}$, and $\dot{\mathcal{D}}$ in $H\left(\kappa^{+}\right)^{V}$ for $\mathbb{P}_{\alpha}, p$, and $\mathcal{D}$.

Let us recall the definition of $S$. In $V, S$ is the set of $N$ in $P_{\kappa}\left(H\left(\kappa^{+}\right)\right)$satisfying:

- $N \prec H\left(\kappa^{+}\right)$,

- $\mu+1 \subseteq N$,

- $\kappa_{N}$ is strongly inaccessible,

- $N^{<\kappa_{N}} \subseteq N$,

- $\bar{N} \prec_{1} \bar{H}\left(\kappa_{N}^{+}\right)$.

Since $\kappa$ is weakly compact, the set $S$ is stationary in $V$.

Choose $N$ in $S$ such that the names $\dot{\mathbb{P}}_{\alpha}, \dot{p}$, and $\dot{\mathcal{D}}$ are members of $N$. So $\mathbb{P}_{\alpha}, p$, and $\mathcal{D}$ are in $N[G]$, and $N[G] \prec H\left(\kappa^{+}\right)^{V[G]}$. Let

$$
\sigma: N[G] \rightarrow \overline{N[G]}
$$

be the transitive collapsing map. By Lemmas 6.2, 6.3, and 6.4,

$$
\begin{gathered}
\overline{N[G]}=\bar{N}\left[G \uparrow \kappa_{N}\right] \prec_{1} H\left(\kappa_{N}^{+}\right)^{V\left[G \uparrow \kappa_{N}\right]}, \\
N[G] \cap V=N,
\end{gathered}
$$

and

$$
N[G]^{<\mu} \subseteq N[G]
$$

It follows that

$$
\bar{N}\left[G \uparrow \kappa_{N}\right]^{<\mu} \subseteq \bar{N}\left[G\left\lceil\kappa_{N}\right]\right.
$$

in $V\left[G \uparrow \kappa_{N}\right]$, since $\bar{N}\left[G \uparrow \kappa_{N}\right]$ and $N[G]$ are $\in$-isomorphic.

The forcing poset $\mathbb{P}_{\alpha}$ is $\mu$-closed, so $N[G]$ models that $\mathbb{P}_{\alpha}$ is $\mu$-closed. Therefore $\bar{N}\left[G \uparrow \kappa_{N}\right]$ models that $\sigma\left(\mathbb{P}_{\alpha}\right)$ is $\mu$-closed. But $\bar{N}\left[G \uparrow \kappa_{N}\right]$ is closed under subsets of size less than $\mu$, as noted above. So in $V\left[G \uparrow \kappa_{N}\right], \sigma\left(\mathbb{P}_{\alpha}\right)$ really is $\mu$-closed. Note that

$$
\sigma \uparrow\left(\mathbb{P}_{\alpha} \cap N[G]\right):\left(\mathbb{P}_{\alpha} \cap N[G], \leq\right) \rightarrow\left(\sigma\left(\mathbb{P}_{\alpha}\right), \leq\right)
$$

is an isomorphism.

We apply Theorem 6.1 to the forcing poset $\sigma\left(\mathbb{P}_{\alpha}\right) / \sigma(p)$. Fix a $V\left[G\left\lceil\kappa_{N}\right]\right.$-generic filter $\bar{H}$ on $\sigma\left(\mathbb{P}_{\alpha}\right)$ in $V[G]$, which contains $\sigma(p)$, such that

$$
V[G]=V\left[G \uparrow \kappa_{N}\right][\bar{H}][K],
$$

where $K$ is a $V\left[G \uparrow \kappa_{N}\right][\bar{H}]$-generic filter on some $\mu$-closed forcing poset.

Define $H$ by

$$
H=\sigma^{-1}[\bar{H}] .
$$

So $H$ is a subset of $\mathbb{P}_{\alpha} \cap N[G]$. Note that $p$ is in $H$, any two conditions in $H$ have a lower bound in $H$, and $H$ is $N[G]$-generic for $\mathbb{P}_{\alpha}$. For if $D$ is a dense open subset of $\mathbb{P}_{\alpha}$ in $N[G]$, then $\bar{N}\left[G\left\lceil\kappa_{N}\right]\right.$ models that $\sigma(D)$ is dense open in $\sigma\left(\mathbb{P}_{\alpha}\right)$. The property "dense open" is $\Delta_{0}$, so $\sigma(D)$ is a dense open subset of $\sigma\left(\mathbb{P}_{\alpha}\right)$ in $V\left[G\left\lceil\kappa_{N}\right\rceil\right.$. Since $\bar{H}$ is $V\left[G \uparrow \kappa_{N}\right]$-generic, there is some $s$ in $\bar{H} \cap \sigma(D)$. Then $\sigma^{-1}(s) \in H \cap D \cap N[G]$.

Let us note that

$$
\bigcup\{\operatorname{dom}(s): s \in H\}=N[G] \cap \alpha=N \cap \alpha .
$$


On the one hand, if $s$ is in $H$, then $s$ has domain of size less than $\kappa$, so $\operatorname{dom}(s) \subseteq$ $N[G]$. On the other hand, if $\gamma$ is in $N[G] \cap \alpha$, then by the $N[G]$-genericity of $H$, there is some $s$ in $H$ with $\gamma \in \operatorname{dom}(s)$.

Define a partial function $q: \alpha \rightarrow X$ with domain $N[G] \cap \alpha$ as follows. By the compatibility of conditions in $H$ and the $N[G]$-genericity of $H$, for each $\beta$ in $N[G] \cap \alpha$, the set

$$
\bigcup\{s(\beta): s \in H\}
$$

is cofinal in $\kappa_{N}$, and is closed below $\kappa_{N}$. For each $\beta$ in $N[G] \cap \alpha$, define

$$
q(\beta)=\bigcup\{s(\beta): s \in H\} \cup\left\{\kappa_{N}\right\} .
$$

Then $q$ is a partial function $q: \alpha \rightarrow X$, with domain of size $|N[G] \cap \alpha| \leq|N[G]|=$ $\mu<\kappa$.

If $s$ is in $H$, then $\operatorname{dom}(s) \subseteq \operatorname{dom}(q)$, and for all $\beta$ in $\operatorname{dom}(s), q(\beta) \leq_{X} s(\beta)$. So if we can show that $q$ is a condition in $\mathbb{P}_{\alpha}$, then $q \leq s$ in $\mathbb{P}_{\alpha}$ for all $s$ in $H$. This implies $q \leq p$ and $q \in \bigcap \mathcal{D}$. For let $D$ be in $\mathcal{D}$. Since $\mathcal{D} \in N[G]$ and $|\mathcal{D}|=\mu<\kappa$, $\mathcal{D} \subseteq N[G]$. Hence $D \in N[G]$. Therefore we can fix some $s$ in $D \cap H$. Then $q \leq s$, so $q \in D$, as $D$ is open. Thus we will be done if we can show that $q$ is in $\mathbb{P}_{\alpha}$.

Let us suppose for a contradiction that $q$ is not a condition in $\mathbb{P}_{\alpha}$. By Lemma 6.9 , there is $\nu$ in $\operatorname{dom}(q)$ such that $q \uparrow \nu$ is in $\mathbb{P}_{\nu}$, but $q \uparrow \nu$ does not force that $q(\nu)$ is disjoint from $\dot{T}(\nu)$. In particular, $\nu$ is in $N[G]$.

Let

$$
H_{\nu}=\{s \uparrow \nu: s \in H\}
$$

Then it is easy to see that

$$
H_{\nu}=H \cap \mathbb{P}_{\nu},
$$

and moreover, $H_{\nu}$ is $N[G]$-generic for $\mathbb{P}_{\nu}$. Also, since $q \uparrow \nu$ is a condition in $\mathbb{P}_{\nu}$, $q \uparrow \nu$ is clearly a lower bound of $H_{\nu}$.

Fix a $\mathbb{P}_{\nu}$-name $\dot{\mathcal{C}}$ in $N[G]$ for a partial square sequence with domain $\dot{T}(\nu)$. For each $\xi$ in $\kappa$, let $\dot{c}_{\xi}$ be a $\mathbb{P}_{\nu}$-name such that $\mathbb{P}_{\nu}$ forces:

$$
\dot{c}_{\xi}= \begin{cases}\dot{\mathcal{C}}(\xi) & \text { if } \xi \in \dot{T}(\nu) \\ \emptyset & \text { if } \xi \notin \dot{T}(\nu)\end{cases}
$$

where $\dot{\mathcal{C}}(\xi)$ is the club indexed by $\xi$ in $\dot{\mathcal{C}}$. By elementarity, there is such a sequence of names $\left\langle\dot{c}_{\xi}: \xi \in \kappa\right\rangle$ in $N[G]$.

For each $\gamma$ in $\kappa$, let $\dot{d}_{\gamma}$ be a $\mathbb{P}_{\nu}$-name such that $\mathbb{P}_{\nu}$ forces:

$$
\dot{d}_{\gamma}= \begin{cases}\dot{c}_{\xi} \cap \gamma & \text { if } \xi \in \dot{T}(\nu) \text { and } \gamma \in \lim \dot{c}_{\xi} \\ \emptyset & \text { if there is no such } \xi\end{cases}
$$

Note that by coherence, the definition of $\dot{d}_{\gamma}$ in the first case is independent of the choice of $\xi$. By elementarity, there is such a sequence of names $\left\langle\dot{d}_{\xi}: \xi \in \kappa\right\rangle$ in $N[G]$.

Now each $\dot{d}_{\gamma}$ is forced by $\mathbb{P}_{\nu}$ to be a set of ordinals of order type less than $\mu$. Since $\mathbb{P}_{\nu}$ is $\mu$-closed, each $\dot{d}_{\gamma}$ is forced to be in the ground model. For each $\gamma$ in $\kappa_{N}$, let

$$
d_{\gamma}=v
$$

where $v$ is a set such that for some $t$ in $H_{\nu}$,

$$
t \Vdash \dot{d}_{\gamma}=v .
$$


Note that by the $N[G]$-genericity of $H_{\nu}, d_{\gamma}$ is defined for all $\gamma$ in $\kappa_{N}$, and by elementarity each $d_{\gamma}$ is in $N[G]$. This defines a sequence

$$
\left\langle d_{\gamma}: \gamma \in \kappa_{N}\right\rangle
$$

in the model $V[G]$.

Recall that $q \uparrow \nu$ does not force that $q(\nu)$ is disjoint from $\dot{T}(\nu)$. But every proper initial segment of $q(\nu)$ is an initial segment of $s(\nu)$, for some $s$ in $H$, and hence is forced by $q \uparrow \nu$ to be disjoint from $\dot{T}(\nu)$. So there is an extension of $q \uparrow \nu$ which forces that $\kappa_{N}$ is in $\dot{T}(\nu)$.

Now $\dot{c}_{\kappa_{N}}$, if non-empty, has order type $\mu$, and by assumption, $\mathbb{P}_{\nu}$ is $\kappa$-distributive. So we can find $r \leq q \uparrow \nu$ and a club $c \subseteq \kappa_{N}$ of order type $\mu$ such that

$$
r \Vdash \dot{c}_{\kappa_{N}}=c .
$$

It follows that

$$
\forall \gamma \in \lim c \quad r \Vdash \dot{d}_{\gamma}=c \cap \gamma .
$$

But $r$ is a lower bound of $H_{\nu}$. Hence

$$
\forall \gamma \in \lim c \quad r \Vdash d_{\gamma}=c \cap \gamma .
$$

Therefore

$$
\forall \gamma \in \lim c \quad d_{\gamma}=c \cap \gamma
$$

In other words, $c$ is a thread of the sequence $\left\langle d_{\gamma}: \gamma \in \kappa_{N}\right\rangle$ which is in $V[G]$.

Let

$$
\bar{H}_{\nu}=\{t \uparrow \sigma(\nu): t \in \bar{H}\}
$$

Then

$$
\bar{H}_{\nu}=\bar{H} \cap \sigma\left(\mathbb{P}_{\nu}\right)
$$

Also

$$
\bar{H}_{\nu}=\sigma\left[H_{\nu}\right]
$$

By 6.7(3) applied to $\sigma\left(\mathbb{P}_{\alpha}\right)$ and $\sigma\left(\mathbb{P}_{\nu}\right)$, it is easy to check that $\bar{H}_{\nu}$ is a $V\left[G \uparrow \kappa_{N}\right]$ generic filter on $\sigma\left(\mathbb{P}_{\nu}\right)$.

We can factor $\sigma\left(\mathbb{P}_{\alpha}\right)$ as

$$
\sigma\left(\mathbb{P}_{\nu}\right) * \sigma\left(\mathbb{P}_{\alpha} / \mathbb{P}_{\nu}\right)
$$

and $\sigma\left(\mathbb{P}_{\nu}\right)$ forces $\sigma\left(\mathbb{P}_{\alpha} / \mathbb{P}_{\nu}\right)$ is $\mu$-closed. More specifically, $\bar{N}\left[G \uparrow \kappa_{N}\right]$ models these facts; but the $<\mu$-closure of $\bar{N}\left[G \uparrow \kappa_{N}\right]$ easily implies they are true in $V\left[G \uparrow \kappa_{N}\right]$. Hence we can write

$$
V[G]=V\left[G \uparrow \kappa_{N}\right]\left[\bar{H}_{\nu}\right]\left[\bar{H}_{\nu, \alpha}\right][K],
$$

where $\bar{H}_{\nu, \alpha}$ is a generic filter for the $\mu$-closed forcing $\sigma\left(\mathbb{P}_{\alpha} / \mathbb{P}_{\nu}\right)$. So $V[G]$ is a generic extension of $V\left[G \uparrow \kappa_{N}\right]\left[\bar{H}_{\nu}\right]$ by a $\mu$-closed forcing.

Let us prove that the sequence $\left\langle d_{\gamma}: \gamma<\kappa_{N}\right\rangle$ is in $V\left[G \uparrow \kappa_{N}\right]\left[\bar{H}_{\nu}\right]$. Note that

$$
\sigma\left(\left\langle\dot{d}_{\gamma}: \gamma \in \kappa\right\rangle\right)=\left\langle\sigma\left(\dot{d}_{\gamma}\right): \gamma \in \kappa_{N}\right\rangle .
$$

For $\gamma$ in $\kappa_{N}$, there is $s$ in $H_{\gamma}$ such that $s \Vdash \dot{d}_{\gamma}=d_{\gamma}$. Then $\sigma(s) \in \bar{H}_{\nu}$, and

$$
\sigma(s) \Vdash \sigma\left(\dot{d}_{\gamma}\right)=\sigma\left(d_{\gamma}\right) .
$$

(Recall that this last forcing statement is absolute between $\bar{N}\left[G \uparrow \kappa_{N}\right]$ and $V[G \uparrow$ $\left.\kappa_{N}\right]$.) But $d_{\gamma}$ is a bounded subset of $\kappa_{N}$, and the critical point of $\sigma^{-1}$ equals $\kappa_{N}$. Hence $\sigma\left(d_{\gamma}\right)=d_{\gamma}$. So

$$
\sigma(s) \Vdash \sigma\left(\dot{d}_{\gamma}\right)=d_{\gamma}
$$


It follows that

$$
\sigma\left(\dot{d}_{\gamma}\right)^{\bar{H}_{\nu}}=d_{\gamma}
$$

Now the sequence $\left\langle\sigma\left(\dot{d}_{\gamma}\right): \gamma<\kappa_{N}\right\rangle$ and the set $\bar{H}_{\nu}$ are in $V\left[G \uparrow \kappa_{N}\right]\left[\bar{H}_{\nu}\right]$. So the sequence $\left\langle d_{\gamma}: \gamma<\kappa_{N}\right\rangle$ is in $V\left[G\left\lceil\kappa_{N}\right]\left[\bar{H}_{\nu}\right]\right.$.

Recall that $\kappa_{N}$ is equal to $\mu^{+}$in the model $V\left[G \uparrow \kappa_{N}\right]$. We claim that $\kappa_{N}$ equals $\mu^{+}$in the model $V\left[G \uparrow \kappa_{N}\right]\left[\bar{H}_{\nu}\right]$. It suffices to show that $\sigma\left(\mathbb{P}_{\nu}\right)$ is $\kappa_{N}$-distributive in $V\left[G \uparrow \kappa_{N}\right]$. The forcing poset $\mathbb{P}_{\nu}$ is $\kappa$-distributive in $V[G]$, so

$$
N[G] \models \mathbb{P}_{\nu} \text { is } \kappa \text {-distributive. }
$$

As $\sigma$ is an isomorphism,

$$
\bar{N}\left[G\left\lceil\kappa_{N}\right] \models \sigma\left(\mathbb{P}_{\nu}\right) \text { is } \kappa_{N}\right. \text {-distributive. }
$$

But

$$
\bar{N}\left[G \uparrow \kappa_{N}\right] \prec_{1} H\left(\kappa_{N}^{+}\right)^{V\left[G\left\lceil\kappa_{N}\right]\right.},
$$

and the property of being $\kappa_{N}$-distributive is $\Pi_{1}$, so

$$
H\left(\kappa_{N}^{+}\right)^{V\left[G\left\lceil\kappa_{N}\right]\right.} \models \sigma\left(\mathbb{P}_{\nu}\right) \text { is } \kappa_{N} \text {-distributive. }
$$

Since every family of dense open subsets of $\sigma\left(\mathbb{P}_{\nu}\right)$ of size $\mu$ is in $H\left(\kappa_{N}^{+}\right)^{V\left[G \mid \kappa_{N}\right]}$, $\sigma\left(\mathbb{P}_{\nu}\right)$ is $\kappa_{N}$-distributive in $V\left[G \uparrow \kappa_{N}\right]$.

Now we apply Lemma 5.3 to get a contradiction. The model $V[G]$ is a generic extension of $V\left[G \uparrow \kappa_{N}\right]\left[\bar{H}_{\nu}\right]$ by a $\mu$-closed (and hence proper) forcing poset. The sequence $\left\langle d_{\gamma}: \gamma \in \kappa_{N}\right\rangle$ is in $V\left[G \uparrow \kappa_{N}\right]\left[\bar{H}_{\nu}\right]$, and has a thread $c$ in $V[G]$. By Lemma $5.3, c$ is in $V\left[G \uparrow \kappa_{N}\right]\left[\bar{H}_{\nu}\right]$. But this is impossible, because $c$ is a cofinal subset of $\kappa_{N}$ of order type $\mu$, whereas $\kappa_{N}=\mu^{+}$in $V\left[G \uparrow \kappa_{N}\right]\left[\bar{H}_{\nu}\right]$.

Let $\mathbb{P}=\mathbb{P}_{\kappa^{+}}$. This completes the construction of our forcing poset,

$$
\operatorname{CoLL}(\mu,<\kappa) * \mathbb{P} \text {. }
$$

Let $H$ be a $V[G]$-generic filter on $\mathbb{P}$. Since $\mathbb{P}$ is $\kappa$-distributive, $\kappa$ is equal to $\mu^{+}$in $V[G][H]$. Suppose $A$ is a subset of $\mu^{+} \cap \operatorname{cof}(\mu)$ which carries a partial square. Then there is a nice name $\dot{A}$ for $A$ which is forced by $\mathbb{P}$ to carry a partial square. Using the $\kappa^{+}$-chain condition of $\mathbb{P}$ and the fact that $\mathbb{P}=\bigcup_{i<\kappa^{+}} \mathbb{P}_{i}$, we can find $\beta<\kappa^{+}$ such that $\dot{A}$ is a nice $\mathbb{P}_{\beta}$-name for a subset of $\mu^{+} \cap \operatorname{cof}(\mu)$ which carries a partial square (using an argument similar to the proof of 6.14). But then at some stage $\gamma \geq \beta, \kappa \backslash A$ acquires a club subset. Therefore $A$ is non-stationary in $V[G][H]$.

A question which arises from this work is whether a weakly compact cardinal is necessary in the above result, or whether a weaker large cardinal hypothesis suffices. For example, assume $\mu \geq \omega_{1}$ is regular, and there is no stationary subset of $\mu^{+} \cap \operatorname{cof}(\mu)$ which carries a partial square. Does it follow that $\mu^{+}$is weakly compact in $L$ ?

\section{REFERENCES}

[1] J. Baumgartner. Iterated forcing. In Surveys in Set Theory, pages 1-59. Cambridge Univ. Press, 1983.

[2] M. Foreman and S. Todorčević. A new Löwenheim-Skolem theorem. Trans. Amer. Math. Soc., 357(5):1693-1715, 2005.

[3] K. Hauser. Indescribable cardinals and elementary embeddings. J. Symbolic Logic, 56:439-457, 1991.

[4] M. Magidor. Reflecting stationary sets. J. Symbolic Logic, 47(4):755-771, 1982.

[5] H. Sakai. Remark on partial square. Preprint, 2009. 
[6] E. Schimmerling and M. Zeman. Square in core models. Bulletin of Symbolic Logic, 7(3):305314, 2001

[7] S. Shelah. Reflecting stationary sets and successors of singular cardinals. Arch. Math. Logic, $31: 25-53,1991$.

[8] S. Shelah. Proper and Improper Forcing. Perspectives in Mathematical Logic. Springer-Verlag, Berlin, second edition, 1998

Department of Mathematics, University of North Texas

E-mail address: jkrueger@unt.edu 